\title{
ON JORDAN ALGEBRAS OF LINEAR TRANSFORMATIONS
}

BY

\author{
A. A. ALBERT
}

\section{INTRODUCTION}

The set $\mathfrak{M}$ of all linear transformations over a field $\mathfrak{F}$ on an $n$-dimensional linear space $\mathfrak{R}$ over $\mathfrak{F}$ forms a total matric algebra of degree $n$ over $\mathfrak{F}$. Let $\mathfrak{A}$ be any linear subspace over $\mathfrak{F}$ of $\mathfrak{M}$. Then we shall say that $\mathfrak{A}$ is weakly closed with respect to transformation multiplication, that is, the product operation $a b$ of $\mathfrak{M}$, if there exist elements $\lambda$ and $\mu$ not both zero and in $\mathfrak{F}$ such that

$$
\lambda a b+\mu b a
$$

is in $\mathfrak{U}$ for every $a$ and $b$ of $\mathfrak{A}$. It is easy to show that then $\mathfrak{A}$ is necessarily of one of the following three types:

(1) The product ab of any two transformations $a$ and $b$ of $\mathfrak{A}$ is in $\mathfrak{A}$ and $\mathfrak{A}$ is an associative algebra.

(2) The nonassociative product $a \cdot b=a b-b a$ is in $\mathfrak{A}$ for every $a$ and $b$ of $\mathfrak{A}$ and $\mathfrak{A}$ is a Lie algebra.

(3) The characteristic of $\mathfrak{F}$ is not two and $\mathfrak{A}$ is closed with respect to the nonassociative product $a \cdot b=(a b+b a) / 2$. Then $\mathfrak{A}$ forms a commutative nonassociative algebra with respect to the operation $a \cdot b$.

While the investigation of spaces of the first two types is classical( $\left.{ }^{1}\right)$ there has been comparatively little study of algebras of the third type. The study of such algebras seems to have been initiated $\left({ }^{2}\right)$ by $\mathrm{P}$. Jordan in connection with quantum mechanics and we shall call them Jordan algebras. Very little has been known about their general structure except under assumptions of reality conditions $\left({ }^{3}\right)$ which eliminate the possibility of a nonzero radical and

Presented to the Society, February 23, 1946; received by the editors January 2, 1946.

(1) For a bibliography see the author's Structure of algebras, Amer. Math. Soc. Colloquium Publications, vol. 24.

(2) See his papers entitled (1) Über eine Klasse nichtassoziativer hyperkomplexer Algebren, Nachr. Ges. Wiss. Güttingen, 1932, pp. 569-575; (2) Über Verallgemeinerungsmöglichkeiten des Formalismus der Quantenmechanik, Nachr. Ges. Wiss. Göttingen, 1933, pp. 209-214; (3) Über die Multiplikation quantenmechanischer Grössen, Zeitschrift für Physik vol. 80 (1933) pp. 285291.

$\left(^{3}\right)$ See the paper by O. Jordan, J. v. Neumann and E. Wigner entitled $O n$ an algebraic generalization of the guantum mechanical formalism, Ann. of Math. vol. 35 (1934) pp. 29-64, for a discussion of $r$-number systems under the assumption that $a_{1}^{2}+\cdots+a_{t}^{2}=0$ if and only if the $a_{i}$ are all zero. The authors show that all such algebras are direct sums of simple algebras which are indeed Jordan algebras in our sense except for the single case of three-rowed Hermitian matrices of Cayley numbers. See also the second paper in footnote 2 for a discussion of algebras with an involution $a \rightarrow a^{J}$ such that $a_{1} a_{1}^{J}+\cdots+a_{t} a_{t}^{J}=0$ if and only if $a_{1}=\cdots=a_{t}$ $=0$. 
which make the investigation comparatively easy.

In the present paper we shall obtain the fundamental structure theorems for Jordan algebras over a nonmodular field. We shall derive analogues for Jordan algebras of the Lie and Engel theorems on solvable Lie algebras and shall obtain a trace criterion for the existence of a nonzero radical. The results imply a Pierce decomposition for a Jordan algebra relative to an idempotent which is almost precisely that known for any associative algebra. Then semisimple Jordan algebras have unity elements and are direct sums of simple algebras. The center of a simple Jordan algebra is a field and every simple Jordan algebra may be expressed as a central simple algebra over its center. If $\mathfrak{A}$ is central simple every scalar extension $\mathfrak{A}_{\mathfrak{R}}$ of $\mathfrak{A}$ is simple.

We shall also show that if $u$ is any primitive idempotent of a simple algebra $\mathfrak{A}$ the associative product $u \mathfrak{A} u$ is a simple subalgebra of $\mathfrak{A}$ and has $u$ as unity quantity. We shall call $\mathfrak{A}$ a reduced algebra if $\mathfrak{A}$ is simple and $u \mathfrak{A} u$ has order one for every primitive idempotent $u$ of $\mathfrak{A}$. Every central simple algebra has a scalar extension field $\Re$ such that $\mathfrak{A}_{\Omega}$ is a reduced algebra, and we shall determine all reduced Jordan algebras.

\section{Chapter I. General properties}

1. Some properties of linear transformations. Our first chapter will be concerned with the study of tool results for our study of Jordan algebras. We shall include in this study an account of some properties of arbitrary nonassociative algebras.

If $\mathfrak{A}$ is any linear space of linear transformations on a linear space $\mathfrak{R}$ over a field $\mathfrak{F}$ we define $\mathfrak{A}^{k}$ to be the space of all finite sums of products of $k$ elements of $\mathfrak{A}$. The union $\mathfrak{A}^{*}$ of all power spaces $\mathfrak{A}^{k}$ is defined to be the set of all finite sums of finite products of elements of $\mathfrak{A}$. It is an associative subalgebra of the total matric algebra $\mathfrak{M}$ and we call $\mathfrak{A}^{*}$ the enveloping algebra of $\mathfrak{A}$.

If we adjoin the identity transformation $I$ to $\mathfrak{A}^{*}$ we obtain an associative algebra having $I$ as its unity element We shall designate this algebra by $\mathfrak{A} \dagger$. It should be observed that every ideal of $\mathfrak{A}^{*}$ is an ideal of $\mathfrak{A} \dagger$ and that $\mathfrak{A} \dagger$ is not semisimple unless $\mathfrak{H}^{*}$ is semisimple.

We shall require the use of

Lemma 1 Let $\mathfrak{A}$ be a linear space of linear transformations and $\mathfrak{A}^{*}$ be nilpotent. Then there exists a singular idempotent linear transformation e such that $e a=a$ for every $a$ of $\mathfrak{A}$.

For let $\mathfrak{N}=\mathfrak{Y}^{*}$ be nilpotent of index $k$. Then $\mathfrak{N}^{k}=0, \mathfrak{N}^{k-1} \neq 0$ and the linear subspace $\mathfrak{R}_{0}=\mathfrak{R} \mathfrak{R}^{k-1}$ of the space $\mathfrak{R}$ on which the transformations of $\mathfrak{A}$ operate has the property that $\mathfrak{R}_{0} \mathfrak{N}=0$. Since $\mathfrak{R}^{k-1} \neq 0$ we have $\mathfrak{R}_{0} \neq 0$. Also $\mathfrak{R}_{0} \neq \mathfrak{R}$ since $\mathfrak{R}_{0} \mathfrak{N}=0$, but $\mathfrak{R} \mathfrak{N} \neq 0$ unless $\mathfrak{N}=0$. It follows that there exists a singular idempotent linear transformation $g$ whose rank $r$ is the order of $\mathfrak{R}_{0}$, such that $\mathfrak{R}_{0}=\mathfrak{R} g$. Then $(x g) h=0$ for every $h$ of $\mathfrak{R}$ and $x$ of $\mathfrak{R}, g h=0$. Write $I=e+g$ where 
$e$ and $g$ are then orthogonal idempotents whose sum is the identity transformation $I$. Then $e h=(I-g) h=h-g h=h$ for every $h$ of $\mathfrak{N}$, and $e a=a$ for every $a$ of $\mathfrak{A}$ as desired.

2. The multiplications of an algebra. The right multiplications of an arbitrary algebra $\mathfrak{A}$ over a field $\mathfrak{F}$ are the linear transformations $R_{x}$ defined by $a \cdot x=a R_{x}$ for every $a$ and $x$ where $a \cdot x$ is the product $\left({ }^{4}\right)$ defining $\mathfrak{A}$. Similarly the left multiplications $L_{x}$ are the linear transformations defined by $x \cdot a=a L_{x}$.

If $\mathfrak{B}$ is any linear subspace of $\mathfrak{A}$ the right multiplications $R_{b}$ determined by the elements $b$ of $\mathfrak{B}$ span a linear subspace $R(\mathfrak{B})$ of the total matric algebra $\mathfrak{M}$ of all linear transformations on $\mathfrak{A}$. Similarly the left multiplications $L_{b}$ span a subspace $L(\mathfrak{B})$. The sum $\mathfrak{Q}=R(\mathfrak{B})+L(\mathfrak{B})$ has an enveloping algebra $E(\mathfrak{B})=\mathfrak{Q}^{*}$ and we extend $E(\mathfrak{B})$ to the transformation algebra $T(\mathfrak{B})=\mathfrak{Q} \dagger$ by adjoining $I$. We may now prove

THEOREM 1. Let $E(\mathfrak{H})$ have a unity quantity $J$. Then $J$ is the identity transformation $I$ on $\mathfrak{A}$ if and only if $\mathfrak{A}$ contains no absolute divisors of zero. When $J \neq I$ the algebra $\mathfrak{A}$ is the direct sum $\mathfrak{B} \oplus \mathfrak{S}$ of its ideal $\mathfrak{B}=\mathfrak{A}(I-J)$ of all absolute divisors of zero in $\mathfrak{A}$ and its ideal $\mathfrak{E}=\mathfrak{A} J$ which has the property that $E(\mathfrak{E})=E(\mathfrak{A})$;

For we define $\mathfrak{B}$ to be the set of all elements $b$ of $\mathfrak{A}$ such that $b J=0$. If $b$ is an absolute divisor of zero in $\mathfrak{A}$ then $x \cdot b=b L_{x}=0, b \cdot x=b R_{x}=0$ for every $x$ of $\mathfrak{A}$. But then $b S=0$ for every $S$ of $E(\mathfrak{A}), b J=0, b$ is in $\mathfrak{B}$. Conversely if $b J=0$ then $b R_{x}=b J R_{x}=0, b L_{x}=b J L_{x}=0$ and $b$ is an absolute divisor of zero. Hence $\mathfrak{B}$ consists of all absolute divisors of zero of $\mathfrak{A}$. Evidently $\mathfrak{B}=0$ means that $J$ is a nonsingular idempotent linear transformation, that is, $J=I$. Let us suppose then that $\mathfrak{B} \neq 0$ and consequently that $I-J \neq 0$. We may now write every $a$ of $\mathfrak{A}$ in the form $a=a(I-J)+a J=b+c$ where $b=a(I-J)$ has the property that $b J=a\left(I J-J^{2}\right)=a(J-J)=0, b$ is in $\mathfrak{B}, c=a J=c J$. It should be clear that the linear subspaces $\mathfrak{A}(I-J)$ and $\mathfrak{E}=\mathfrak{A} J$ of $\mathfrak{A}$ are supplementary and that $\mathfrak{B}=\mathfrak{A}(I-J)$. Now $b \cdot c=c \cdot b=0$ for every $b$ of $\mathfrak{B}$ and $c$ of $\mathfrak{E}$ since every $b \cdot x=x \cdot b=0$. If $b$ is in $\mathfrak{B}$ then $R_{b}=0$ and $R_{a}=R_{b+c}=R_{c}$. Let $x=y+z$ where $y$ is in $\mathscr{B}$ and $z$ is in $\left(\mathcal{C}\right.$. Then $a \cdot x=c \cdot z=c R_{z}=c R_{z} J$ since $J$ is the identity element of $E(\mathfrak{H})$ and all $R_{x}$ are in $E(\mathfrak{H})$. Then $a \cdot x=c \cdot z$ is in $\mathfrak{C}$ for every $a$ and $x$ of $\mathfrak{A}$. This proves that $\mathfrak{E}$ is an ideal of $\mathfrak{A}$ and implies that $\mathfrak{A}=\mathfrak{B} \oplus \mathfrak{E}$. Evidently $R(\mathfrak{U})=R(\mathfrak{S}), L(\mathfrak{U})=L(\mathfrak{S}), E(\mathfrak{I})=E(\mathfrak{S})$.

The result above is required $\left(^{(5)}\right.$ in the study of algebras homomorphic to semisimple algebras. An algebra $\mathfrak{A}$ is said to be simple if it has no ideals except $\mathfrak{A}$ and the zero ideal and if $\mathfrak{A}$ is not a zero algebra of order one. An algebra $\mathfrak{A}$

(4) In our discussion of Jordan algebras we shall always use the notations $a b, a \mathfrak{B}, \mathfrak{B}^{2}, \mathfrak{B C}$ to indicate associative products of elements $a, b$ and of spaces $\mathfrak{B}, \mathfrak{C}$, and $a \cdot b, a \cdot \mathfrak{B}, \mathfrak{B} \cdot \mathfrak{C}, \mathfrak{B} \cdot \mathbf{2}$ to indicate the Jordan products.

(5) This result is needed in the proof of Lemma 2 in the author's paper The radical of $a$ nonassociative algebra, Bull. Amer. Math. Soc. vol. 48 (1942) pp. 891-897. We shall complete the correction of the error due to its omission by proving the present Theorem 2. 
is said to be semisimple if $\mathfrak{A}$ is the direct sum of simple algebras. Let us now assume that $\mathfrak{A}$ is any algebra such that $T(\mathfrak{H})$ is semisimple.

The radical of the associative algebra $E(\mathfrak{A})$ is a nilpotent ideal of $T(\mathfrak{H})$ and so when $T(\mathfrak{H})$ is semisimple so is $E(\mathfrak{H})$. Then $E(\mathfrak{H})$ has a unity element $J$. By Theorem 1 we see that $J=I$ if and only if $\mathfrak{A}$ contains no absolute divisors of zero. In this case $T(\mathfrak{H})=E(\mathfrak{A})$ and it is known $\left({ }^{6}\right)$ that $\mathfrak{A}$ is semisimple. If, however, $T(\mathfrak{H}) \neq E(\mathfrak{H})$ then $J \neq I, \mathfrak{A}=\mathfrak{E} \oplus \mathfrak{B}, \mathfrak{E}=\mathfrak{A} J$ has the property that $E(\mathfrak{C})=E(\mathfrak{H})$. Moreover the transformations of $E(\mathfrak{C})$ may be regarded as linear transformations on the subspace $\mathfrak{E}=\mathfrak{A} J$ of $\mathfrak{A}$ and the identity transformation of this space is the unity element $J$ of $E(\mathbb{E})$, $\mathfrak{C}$ is semisimple. Also $\mathfrak{B}=\mathfrak{A}(I-J)$ is a zero algebra and we have

THEOREM 2. The transformation algebra $T(\mathfrak{A})$ is semisimple if and only if $\mathfrak{A}$ is either semisimple, so that $E(\mathfrak{H})=T(\mathfrak{A})$, or $\mathfrak{A}=\mathfrak{B} \oplus \mathbb{C}$ where $\mathfrak{B}$ is a zero algebra, $\mathfrak{C}$ is semisimple, and $E(\mathfrak{A}) \neq T(\mathfrak{A})$.

3. Difference algebras. In this section we shall give a brief repetition of some well known concepts of algebra, calling attention to the particular form in which we shall use the concepts.

If $\mathfrak{A}$ is an algebra and $\mathfrak{B}$ is an ideal of $\mathfrak{A}$ we define the class $[a]$, for every $a$ of $\mathfrak{A}$, to be the set of all elements $a+b$ with $b$ in $\mathfrak{B}$. Then the operations $[a]+[b]=[a+b],[a] \cdot[b]=[a \cdot b]$ define an algebra $\mathfrak{A}-\mathfrak{B}$ whose elements are the classes $[a]$ and which is called the difference algebra of $\mathfrak{A}$ modulo $\mathfrak{B}$. The mapping $a \rightarrow[a]$ is then a homomorphism of $\mathfrak{A}$ on $\mathfrak{A}-\mathfrak{B}$.

If $a \rightarrow a_{0}$ is any homomorphism of $\mathfrak{A}$ on an algebra $\mathfrak{A}_{0}$ the set $\mathfrak{B}$ of all elements $b$ of $\mathfrak{A}$ such that $b_{0}=0$ is an ideal of $\mathfrak{A}$. Moreover the mapping $[a] \rightarrow a_{0}$ is an isomorphism of $\mathfrak{A}-\mathfrak{B}$ and $\mathfrak{A}_{0}$. The homomorphism $a \rightarrow a_{0}$ maps every subalgebra $\mathfrak{E}$ of $\mathfrak{A}$ homomorphically on a subalgebra $\mathfrak{E}_{0}$ of $\mathfrak{A}_{0}$. Then we shall require the following result:

Lemma 2. Let $\mathfrak{D}$ be the intersection of $\mathfrak{C}$ and $\mathfrak{B}$. Then $\mathfrak{D}$ is an ideal of $\mathfrak{C}$, $\mathfrak{C}-\mathfrak{D}$ is isomorphic to $\mathfrak{S}_{0}$.

Indeed if $\{c\}$ is the class of all elements of $\mathbb{E}$ of the form $c+d$ with $d$ ranging over elements of $\mathfrak{D}$, and $c$ maps into $c_{0}$, then $\{c\} \rightarrow c_{0}$ is readily seen to be an isomorphism of $\mathfrak{E}-\mathfrak{D}$ and $\mathfrak{S}_{0}$.

4. Solvable algebras. The fact that every nonassociative algebra contains a maximal solvable ideal requires the proof of a theorem. This can be supplied not only for Lie and Jordan algebras but for all nonassociative algebras.

If $\mathfrak{A}$ is any nonassociative algebra we define the derived series of $\mathfrak{A}$ to be the sequence of subalgebras (or zero subspaces) of $\mathfrak{A}$ defined recursively according to

$$
\mathfrak{A}^{(0)}=\mathfrak{A}, \mathfrak{A}^{(1)}=\mathfrak{A}^{\cdot 2}, \mathfrak{A}^{(2)}=\left[\mathfrak{H}^{(1)}\right] \cdot 2, \cdots, \mathfrak{A}^{(k)}=\left[\mathfrak{H}^{(k-1)}\right] \cdot 2, \cdots,
$$

(6) The proof is given on the top of page 894 in the paper referred to in footnote 5. 
where $\mathfrak{A} \cdot \boldsymbol{2}$ is defined to be the linear space of all finite sums of products $a \cdot b$ for $a$ and $b$ in $\mathfrak{A}$. We call $\mathfrak{A}$ a solvable algebra of index $k$ if there exists an integer $k>0$ such that $\mathfrak{A}^{(k-1)} \neq 0, \mathfrak{P}^{(k)}=0$.

An ideal $\mathfrak{B}$ of an algebra $\mathfrak{A}$ is called a solvable ideal of $\mathfrak{A}$ if $\mathfrak{B}$ is either zero or a solvable algebra. We may then prove

Lemma 3. Let $\mathfrak{B}$ be a solvable ideal of $\mathfrak{A}$ and $\mathfrak{A}-\mathfrak{B}$ be solvable. Then $\mathfrak{A}$ is solvable.

For the hypothesis that $\mathfrak{A}-\mathfrak{B}$ is solvable is precisely that there exists an in teger $k$ such that $(\mathfrak{A}-\mathfrak{B})^{(k)}=0$, that is, $\mathfrak{A}^{(k)} \leqq \mathfrak{B}$. Then $\mathfrak{A}^{(k+s)} \leqq \mathfrak{B}^{(s)}$ for every $s$ and the existence of an $s$ such that $\mathfrak{B}^{(s)}=0$ implies that $\mathfrak{A}^{(k+s)}=0, \mathfrak{A}$ is solvable.

If $\mathfrak{B}$ is any subalgebra of our algebra $\mathfrak{A}$ the property that $\mathfrak{B}^{(s)} \leqq \mathfrak{A}^{(s)}$ is trivial. Hence we have

Lemma 4. Every subalgebra of a solvable algebra is solvable.

The sum $\mathfrak{B}=\mathfrak{B}+\mathfrak{C}$ of any two ideals of an algebra $\mathfrak{A}$ is trivially seen to be an ideal of $\mathfrak{A}$. The mapping of $\mathfrak{A}$ on $\mathfrak{A}-\mathfrak{B}$ maps $\mathfrak{B}+\mathfrak{C}$ homomorphically on a subalgebra $\mathfrak{B}-\mathfrak{B}$ of $\mathfrak{A}-\mathfrak{B}$ and it should be obvious that $\mathfrak{E}$ is homomorphic to $(\mathfrak{S}-\mathfrak{B}$. Then $\mathbb{B}-\mathfrak{B}$ is solvable. By Lemma 3 , BS is solvable. It follows that the sum of two solvable ideals of an algebra is solvable and we may draw the conclusion stated as

THEOREM 3. Every solvable ideal of any algebra $\mathfrak{A}$ is an ideal contained in the unique maximal solvable ideal $\mathfrak{N}$ of $\mathfrak{A}$. The only solvable ideal of $\mathfrak{A}-\mathfrak{N}$ is the zero ideal.

5. Nilpotent algebras. Every sequence $a_{1}, \cdots, a_{k}$ of $k$ elements of an algebra $\mathfrak{A}$ defines a special product $a^{(k)}$ of order $k$ by the formula $a^{(i)}=a^{(i-1)} \cdot a_{i}$ or $a^{(i)}=a_{i} \cdot a^{(i-1)}$ for $i>1$. Then we shall say that $\mathfrak{A}$ is nilpotent of index $k$ if all special products of order $k$ are zero and some special product of order $k-1$ is not zero.

An algebra $\mathfrak{A}$ is said to be strongly nilpotent if there exists an integer $k$ such that all products of $k$ elements of $\mathfrak{A}$ are zero. A strongly nilpotent algebra is evidently both nilpotent and solvable. We shall prove that in the case of Jordan algebras the concepts of solvability, nilpotency, and strong nilpotency are equivalent.

We may now prove

Lemma 5. An ideal $\mathfrak{B}$ of $\mathfrak{A}$ is nilpotent if and only if $E(\mathfrak{B})$ is nilpotent.

For every special product $a^{(k+1)}$ defined by a sequence $a_{1}, \cdots, a_{k+1}$ with $a_{2}, \cdots, a_{k+1}$ in $\mathfrak{B}$ is also a special product of $k$ elements $b_{1}=a_{1} \cdot a_{2}$ or $a_{2} \cdot a_{1}$, $b_{2}=a_{3}, \cdots, b_{k}=a_{k+1}$ in $\mathfrak{B}$. Then $a^{(k+1)}=0$ for every $x=a_{1}$ in $\mathfrak{A}$. But $a^{(k+1)}=x S_{1} \cdots S_{k}$ where $S_{1}, \cdots, S_{k}$ are arbitrary elements of $R(\mathfrak{B})+L(\mathfrak{B})$. 
It follows that every $S_{1} \cdots S_{k}=0, E(B)$ is nilpotent of index at most $k$. Conversely if $E(\mathfrak{B})$ is nilpotent then all special products of order $k+1 \mathrm{de}$ fined for elements in $\mathfrak{B}$ have the form $b S_{1} \cdots S_{k}$ and are zero, $\mathfrak{B}$ is nilpotent.

It does not seem possible to prove that if $\mathfrak{B}$ is a nilpotent ideal of $\mathfrak{A}$ the nilpotent algebra $E(\mathfrak{B})$ is always contained in the radical of the associative algebra $E(\mathfrak{R})$ although this result is true for Lie algebras and Jordan algebras. It also does not seem evident that the sum of two nilpotent ideals is nilpotent nor even that the sum of two strongly nilpotent ideals is nilpotent.

6. The ideals of a semisimple algebra. If $\mathfrak{A}$ is any algebra its square $\mathfrak{A} \cdot 2$ is an ideal of $\mathfrak{A}$. If $\mathfrak{A}$ is simple it is not a zero algebra, $\mathfrak{A} \cdot 2 \neq 0$, and so $\mathfrak{A} \cdot{ }^{\cdot 2}=\mathfrak{A}$. If $\mathfrak{A}=\mathfrak{A}_{1} \oplus \cdots \oplus \mathfrak{A}_{t}$ is the semisimple direct sum of the simple algegras $\mathfrak{A}_{i}$ then each $\mathfrak{A}_{i} \cdot 2=\mathfrak{A}_{i}$ and $\mathfrak{A} \cdot 2 \geqq \mathfrak{A}_{i} \cdot{ }^{2}=\mathfrak{A}_{i}, \mathfrak{A} \cdot 2 \geqq \mathfrak{A}, \mathfrak{A} \cdot 2=\mathfrak{A}$.

The ideals of semisimple algebra with a unity quantity have been shown $\left({ }^{7}\right)$ to have the property of the following theorem but we can show that the result holds generally.

THEOREM 4. Let $\mathfrak{B}$ be an ideal of a semisimple algebra $\mathfrak{A}=\mathfrak{A}_{1} \oplus \cdots \oplus \mathfrak{A}_{t}$, where the components $\mathfrak{A}_{i}$ are simple algebras, $\mathfrak{B}_{i}$ be the intersection of $\mathfrak{A}_{i}$ and $\mathfrak{B}$. Then $\mathfrak{B}_{\mathfrak{i}}=0$ or $\mathfrak{A}_{i}, \mathfrak{B}$ is the semisimple direct sum of those components $\mathfrak{A}_{i}$ for which $\mathfrak{B}_{i} \neq 0, \mathfrak{A}=\mathfrak{B} \oplus \mathfrak{C}, \mathfrak{C}$ is the direct sum of those components $\mathfrak{A}_{i}$ such that $\mathfrak{B}_{i}=0$.

For every $b$ of $\mathfrak{B}$ has the form $b=b_{1}+\cdots+b_{t}$ with $b_{i}$ in $\mathfrak{A}_{i}$. Now $a_{i} \cdot b$ and $b \cdot a_{i}$ are in $\mathfrak{B}_{i}$ for every $a_{i}$ of the ideal $\mathfrak{A}_{i}$ and every $b$ of $\mathfrak{B}$. But $a_{i} \cdot b=a_{i} \cdot b_{i}$, $b \cdot a_{i}=b_{i} \cdot a_{i}$ and it follows that the set $\mathfrak{E}_{i}$ of all components $b_{i}$ of every $b$ of $\mathfrak{B}$ has the property that $\mathfrak{A}_{i} \cdot \mathfrak{C}_{i} \leqq \mathfrak{B}_{i}, \mathfrak{C}_{i} \cdot \mathfrak{A}_{i} \leqq \mathfrak{B}_{i}$. Clearly $\mathfrak{A}_{i} \geqq \mathfrak{C}_{i} \geqq \mathfrak{B}_{i}$ and we have proved that $\mathfrak{B}_{i}$ and $\mathfrak{S}_{i}$ are ideals of $\mathfrak{A}_{i}$. If $\mathfrak{S}_{i}=0$ then $\mathfrak{B}_{i}=0$ and no $b$ of $\mathfrak{B}$ has a nonzero component in $\mathfrak{A}_{i}$. Otherwise $\mathfrak{\mathfrak { V }}_{i}=\mathfrak{A}_{i}$ and the definition of $\mathfrak{E}_{i}$ implies that $\mathfrak{C}_{i} \cdot \mathfrak{B}=\mathfrak{C}_{i} \cdot 2=\mathfrak{A}_{i} \cdot 2=\mathfrak{A}_{i} \leqq \mathfrak{B}_{i}, \mathfrak{B}_{i}=\mathfrak{C}_{i}$. This proves that the elements of $\mathfrak{B}$ are the sums of elements in subalgebras $\mathfrak{A}_{i}$ of $\mathfrak{B}$ and thus that $\mathfrak{B}$ is the direct sum of these subalgebras. The remainder of the proof is trivial.

The theorem above implies that the components in the expression of a semisimple algebra as a direct sum of simple components are unique. For if $\mathfrak{B}$ is a component it is a simple ideal of $\mathfrak{A}$ and is one of the $\mathfrak{A}_{i}$.

We have already seen that $\mathfrak{A} \cdot 2=\mathfrak{A}$ when $\mathfrak{A}$ is semisimple. Every ideal $\mathfrak{B}$ of $\mathfrak{A}$ is semisimple and $\mathfrak{B}^{\cdot 2}=\mathfrak{B}$. Then $\mathfrak{B}$ cannot be solvable and we have

THeOREM 5. The maximum solvable ideal of a semisimple algebra is the zero ideal.

7. The radical of an algebra. If an algebra $\mathfrak{A}$ is homomorphic to a semisimple algebra there exists $\left({ }^{8}\right)$ an ideal $\mathfrak{N}$ called the radical of $\mathfrak{A}$ such that

( $\left.{ }^{7}\right)$ See Lemma 5 of the author's Nonassociative algebras I: New simple alyebras, Ann. of Math. vol. 43 (1942) pp, 708-723.

${ }^{(8)}$ See the paper referred to in footnote 5. 
$\mathfrak{A}-\mathfrak{N}$ is semisimple, $\mathfrak{N}$ is contained in every ideal $\mathfrak{B}$ such that $\mathfrak{A}-\mathfrak{B}$ is semisimple. We now prove

THEOREM 6. The maximal solvable ideal of any algebra $\mathfrak{A}$ is contained in its radical.

For if $\mathfrak{C}$ is a solvable ideal of $\mathfrak{A}$ and $\mathfrak{N}$ is the radical of $\mathfrak{A}$ the mapping of $\mathfrak{A}$ on $\mathfrak{A}-\mathfrak{R}$ maps $\mathfrak{C}$ homomorphically on a subspace $\mathfrak{E}_{0}$ of $\mathfrak{A}-\mathfrak{N}$. Then $\mathfrak{E}_{0}$ is a solvable ideal of $\mathfrak{A}-\mathfrak{N}, \mathfrak{V}_{0}=0$ by Theorem 5 , $\mathfrak{C}$ is contained in $\mathfrak{N}$.

The radical of an algebra need not be its maximal solvable ideal and indeed there exists an example $\left(^{8}\right)$ of an algebra whose radical is an associative field. However we can prove

THEOREM 7. Let $\mathfrak{A}$ be an algebra homomorphic to a semisimple algebra, $\mathfrak{S}$ be the radical of $T(\mathfrak{A})$ and $T(\mathfrak{H})-\mathfrak{S}$ be simple. Then the radical $\mathfrak{N}$ of $\mathfrak{A}$ is a nilpotent ideal of $\mathfrak{A}, \mathfrak{N}$ is the set of all elements $y$ of $\mathfrak{A}$ such that $R_{y}$ and $L_{y}$ are in $\mathfrak{S}, \mathfrak{A}-\mathfrak{N}$ is simple, $E(\mathfrak{N}) \leqq \mathfrak{S}$.

For $\mathfrak{A} \mathfrak{S}=\mathfrak{N}$ is the radical of $\mathfrak{A}$ and there exists a singular idempotent $E$ such that $\mathfrak{N}=\mathfrak{N} E$. Then there is $\left({ }^{8}\right)$ a corresponding ideal $\subseteq$ in $T(\mathfrak{H})$ consisting of all $S=S E$ in $T(\mathfrak{R})$ such that $T(\mathfrak{R})-\mathfrak{S}=T(\mathfrak{A}-\mathfrak{R})$. But $T(\mathfrak{U})-\mathfrak{S}$ is semisimple and $\mathfrak{S}$ contains $\mathfrak{S}$. However the only ideals of $T(\mathfrak{A})$ containing $\mathfrak{S}$ are $T(\mathfrak{A})$ and $\mathfrak{S}$ since $T(\mathfrak{H})-\mathfrak{S}$ is simple. Hence $\mathfrak{S}=\mathfrak{S}$ or $T(\mathfrak{R})$. If $\mathfrak{S}=T(\mathfrak{R})$ then $I E=I=E$ and $\mathfrak{A}=\mathfrak{A} \mathfrak{W}, \mathfrak{A}=\mathfrak{A} \mathfrak{S}^{k}=0$. Hence $\mathfrak{S}=\mathfrak{S}, T(\mathfrak{A}-\mathfrak{N})=T(\mathfrak{A})-\mathfrak{S}$ is simple. Since $\mathfrak{A}$ is homomorphic to a semisimple algebra $\mathfrak{A}-\mathfrak{N}$ is not a zero algebra, $\mathfrak{A}-\mathfrak{N}$ is known $\left(^{9}\right)$ to be simple. If $y$ is in $\mathfrak{N}$ and $x$ is in $\mathfrak{A}$ then $x \cdot y=x R_{y}=(x \cdot y) E=x R_{y} E$ and $R_{y}=R_{y} E, R_{y}$ is in $\mathfrak{S}=\mathfrak{S}$. Similarly $L_{y}$ is in $\mathfrak{S}$, $\mathfrak{N}$ is a subset of the set $\mathfrak{R}_{0}$ of all quantities $y$ of $\mathfrak{A}$ such that $R_{y}$ and $L_{y}$ are in $\mathfrak{S}$. Conversely $\mathfrak{R}_{0}$ is a linear subspace of $\mathfrak{A}$ such that if $x$ is in $\mathfrak{A}$ and $y$ is in $\mathfrak{N}_{0}$ then $x \cdot y=x R_{y}$ and $y \cdot x=x L_{y}$ are in $\mathfrak{N} \leqq \mathfrak{R}_{0}$. Hence $\mathfrak{R}_{0}$ is an ideal of $\mathfrak{A}$. Since $\mathfrak{N}_{0}$ contains $\mathfrak{N}$ we see that $\mathfrak{N}_{0}-\mathfrak{N}$ is an ideal of $\mathfrak{A}-\mathfrak{N}, \mathfrak{N}_{0}-\mathfrak{N}=0$ or $\mathfrak{A}-\mathfrak{N}$, $\mathfrak{N}_{0}=\mathfrak{N}$ or $\mathfrak{A}$. Now $\mathfrak{R}_{0}=\mathfrak{A}$ implies that $E(\mathfrak{A}) \leqq \mathfrak{S}, T(\mathfrak{A})-\mathfrak{S} \rightarrow I \mathfrak{F}$, the homomorphism of $\mathfrak{A}$ and $\mathfrak{A}-\mathfrak{N}$ is such that $[x \cdot y]=[x]\left[R_{y}\right]=0, \mathfrak{A}-\mathfrak{N}$ is a zero algebra. Hence $\mathfrak{N}_{0}=\mathfrak{N}$ as desired.

8. Weakly closed sets of linear transformations. Let $\mathfrak{A}$ be a linear space of linear transformations over $\mathfrak{F}$ and let there exist elements $\lambda$ and $\mu$ in $\mathfrak{F}$ and not both zero such that $c=\lambda a b+\mu b a$ is in $\mathfrak{A}$ for every $a$ and $b$ of $\mathfrak{A}$. Then $\mathfrak{A}$ also contains $d=\lambda b a+\mu a b$ and hence $\mathfrak{A}$ contains

$$
c+d=(\lambda+\mu)(a b+b a), \quad c-d=(\lambda-\mu)(a b-b a) .
$$

If $\lambda \neq \mu,-\mu$ then $\mathfrak{A}$ contains both $a b+b a$ and $a b-b a$. When the characteristic of $\mathfrak{F}$ is not two this implies that $\mathfrak{A}$ contains $a b$ and so is an associative algebra. Evidently it is true conversely that when $\mathfrak{A}$ is an associative algebra

( ${ }^{9}$ See Lemma 10 on page 692 of the author's Nonassociative algebras I: Fundamental concepts and isotopy, Ann. of Math. vol. 43 (1942) pp. 685-708. 
it contains $\lambda a b+\mu b a$ for every $\lambda$ and $\mu$.

Let us now suppose that $\mathfrak{A}$ is not closed with respect to the associative product $a b$. Then either $\mathfrak{A}$ contains $a \cdot b=a b-b a$, or the characteristic of $\mathfrak{F}$ is not two and $\mathfrak{A}$ contains $a b+b a$, or $\lambda= \pm \mu$. In the latter case $\mathfrak{A}$ contains $\lambda(a b \pm b a)$ and again contains $a b+b a$ or $a b-b a$. This completes our determination of the possibilities for $\mathfrak{A}$ and we have proved the result of our introduction.

It should be noted that if $\mathfrak{A}$ is a Lie algebra of linear transformations the right multiplications of $\mathfrak{A}$ satisfy the equation $R_{a \cdot b}=R_{a} R_{b}-R_{b} R_{a}$. However if $\mathfrak{A}$ is a Jordan algebra the right multiplications of $\mathfrak{A}$ do not satisfy the corresponding property

$$
R_{a \cdot b}=\left(R_{a} R_{b}+R_{b} R_{a}\right) / 2 .
$$

For indeed let the law above hold for a commutative algebra $\mathfrak{A}$. Then $R_{a \cdot 2}=\left(R_{a}\right)^{2}$ and $(x \cdot a) \cdot a=x(a \cdot a), a \cdot(a \cdot x)=(a \cdot a) \cdot x, a \cdot(x \cdot a)=x R_{a} L_{a}=x L_{a} R_{a}$ $=(a \cdot x) \cdot a$. Then $\mathfrak{A}$ is a commutative alternative algebra. But the only nonassociative simple alternative algebras are the noncommutative systems with a Cayley number direct factor and so every simple commutative alternative algebra is associative. However the central simple algebras we shall obtain will all be not associative with respect to our operation $a \cdot b=(a b+b a) / 2$.

\section{Chapter II. Jordan Algebras}

1. A fundamental lemma. Let us call a linear space $\mathfrak{A}$ of linear transformations a weakly closed algebra if $\mathfrak{A}$ is an algebra relative to an operation

$$
a \cdot b=\lambda a b+\mu b a \quad(\lambda \mu \neq 0, \lambda, \mu \text { in } \mathfrak{F}) .
$$

Then if $\mathfrak{B}$ is an ideal of $\mathfrak{A}$ we have $a b=b_{1}-\mu \lambda^{-1} b a, b a=b_{2}-\lambda \mu^{-1} a b$. Hence the relations

$$
\mathfrak{U} \mathfrak{B} \leqq \mathfrak{B P}+\mathfrak{B}, \quad \mathfrak{B} \mathfrak{Y} \leqq \mathfrak{A}+\mathfrak{B}
$$

hold where the indicated products are associative products of linear spaces of linear transformations. We may then prove an extension of a lemma of $\mathrm{N}$. Jacobson $\left({ }^{10}\right)$ derived by him for Lie algebras of linear transformations.

Lemma 6. Let $\mathfrak{B}$ be an ideal of a weakly closed algebra $\mathfrak{A}$. Then $\mathfrak{B}^{*} \mathfrak{H}^{*}$ and $\mathfrak{I}^{*} \mathfrak{B}^{*}$ are ideals of $\mathfrak{A}^{*}, \mathfrak{B}^{*} \mathfrak{U} \dagger=\mathfrak{U} \dagger \mathfrak{B}^{*}$ is an ideal of $\mathfrak{U}^{*}, \mathfrak{B}^{*} \mathfrak{U} \dagger \geqq \mathfrak{B}^{*}$. Moreover $\mathfrak{B}^{*} \mathfrak{A}^{*}, \mathfrak{H}^{*} \mathfrak{B}^{*}, \mathfrak{B}^{*} \mathfrak{Y} \dagger$ are nilpotent when $\mathfrak{B}^{*}$ is nilpotent.

For our hypothesis implies the relation

$$
\mathfrak{A} \mathfrak{B}^{*} \leqq \mathfrak{B}^{\text {\& }}+\mathfrak{B}^{\text {* }}
$$

for $s=1$, where $\mathfrak{B}^{s}$ is the associative power. The given relation for a value of $s$

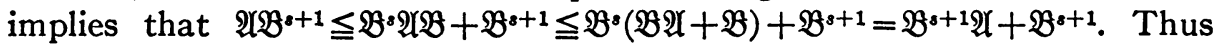

(10) See Lemma 1 of N. Jacobson's Rational methods in the theory of Lie algebras, Ann. of Math. vol. 36 (1935) pp. 875-881. 
(1) holds for all values of $s$ and implies the validity of

$$
\mathfrak{A}^{\mathfrak{t} \mathfrak{B}^{*}} \leqq \mathfrak{B}^{*} \mathfrak{A}^{*}+\mathfrak{B}^{*}
$$

for $t=1$. From this relation for any $t$ we conclude that $\mathfrak{A}^{t+1} \mathfrak{B}^{*} \leqq\left(\mathfrak{H}_{\left.\mathfrak{B}^{*}\right) \mathfrak{A}^{*}}\right.$ $+\mathfrak{A B}^{*} \leqq\left(\mathfrak{B}^{*} \mathfrak{A}^{*}\right) \mathfrak{H}^{*}+\mathfrak{B}^{*} \mathfrak{U}^{*}+\mathfrak{B}^{*} \mathfrak{A}^{*}+\mathfrak{B}^{*} \leqq \mathfrak{B}^{*} \mathfrak{I}^{*}+\mathfrak{B}^{*}$ since $\mathfrak{A}^{*}$ is an associative algebra. This completes our induction and implies the first of the relations

$$
\mathfrak{U}^{*} \mathfrak{B}^{*} \leqq \mathfrak{B}^{*} \mathfrak{I}^{*}+\mathfrak{B}^{*}, \quad \mathfrak{B ^ { * }} \mathfrak{I}^{*} \leqq \mathfrak{I}^{*} \mathfrak{B}^{*}+\mathfrak{B}^{*} .
$$

The second relation follows by symmetry. The relations taken together imply that $\mathfrak{H}^{*} \mathfrak{B}^{*} \mathfrak{H}^{*}$ is contained in the intersection of $\mathfrak{B}^{*} \mathfrak{H}^{*}$ and $\mathfrak{A}^{*} \mathfrak{B}^{*}$. Then $\mathfrak{B}^{*} \mathfrak{I}^{*}$ and $\mathfrak{A}^{*} \mathfrak{B}^{*}$ are ideals of $\mathfrak{A}^{*}$. Now

$$
\mathfrak{A} \dagger=\mathfrak{A}^{*}+\mathfrak{I} \mathfrak{F}
$$

and $\mathfrak{B}^{*} \mathfrak{A} \dagger=\mathfrak{B}^{*} \mathfrak{\mathfrak { A } ^ { * }}+\mathfrak{B}^{*}, \mathfrak{A}+\mathfrak{B}^{*}=\mathfrak{A}^{*} \mathfrak{B}^{*}+\mathfrak{B}^{*}$. By $(3), \mathfrak{B}^{*} \mathfrak{A} \dagger \leqq \mathfrak{A} \dagger \mathfrak{B}^{*}, \mathfrak{A} \dagger \mathfrak{B}^{*} \leqq \mathfrak{B}^{*} \mathfrak{A} \dagger$, $\mathfrak{A}+\mathfrak{B}^{*}=\mathfrak{B}^{*} \mathfrak{A} \dagger \geqq \mathfrak{B}^{*}$. Since $\mathfrak{A} \dagger \mathfrak{B}^{*}$ is contained in $\mathfrak{A}^{*}$ and $\mathfrak{A}^{*}\left(\mathfrak{A}+\mathfrak{B}^{*}\right)=\mathfrak{A}^{*} \mathfrak{B}^{*}$ $+\mathfrak{A}^{*} \mathfrak{B}^{*}=\mathfrak{A}^{*} \mathfrak{B}^{*}, \mathfrak{A} \dagger \mathfrak{B}^{*} \mathfrak{\mathfrak { A } ^ { * }}=\mathfrak{B}^{*} \mathfrak{A}+\mathfrak{A}^{*}=\mathfrak{B}^{*} \mathfrak{A}^{*}$, we see that $\mathfrak{A} \dagger \mathfrak{B}^{*}$ is an ideal of $\mathfrak{A}^{*}$. The equality $\mathfrak{A}+\mathfrak{B}^{*}=\mathfrak{B}^{*} \mathfrak{A} \dagger$ and the associative law imply that $\left(\mathfrak{A}+\mathfrak{B}^{*}\right)^{k}$ $=(\mathfrak{A} \dagger)^{k}\left(\mathfrak{B}^{*}\right)^{k}=0$ if $\left(\mathfrak{B}^{*}\right)^{k}=0$. Then $\mathfrak{A}_{\dagger}+\mathfrak{B}^{*}$ is nilpotent and its subalgebras $\mathfrak{A}^{*} \mathfrak{B}^{*}$ and $\mathfrak{B}^{*} \mathfrak{I}^{*}$ are nilpotent.

Our result implies that when $\mathscr{B} \neq 0$ is an ideal of $\mathfrak{A}$ such that $\mathfrak{B}^{*}$ is nilpotent then $\mathfrak{B}$ is contained in the radical of $\mathfrak{A}^{*}, \mathfrak{A}^{*}$ is not a semisimple algebra. It will be convenient to use this result in the form

Lemma 7. Let $\mathfrak{B}$ be an ideal of a weakly closed algebra $\mathfrak{A}$ such that $\mathfrak{B}^{*}$ is nilpotent. Then $\mathfrak{B}$ and $\mathfrak{B}^{*}$ are contained in the radical of $\mathfrak{A}^{*}$.

2. Solvable Jordan algebras. The results of $\$ 2$ of Chapter I may be used to prove

THEOREM 8. A solvable Jordan algebra $\mathfrak{A}$ of linear transformations is strongly nilpotent and $\mathfrak{A}^{*}$ is the radical of the associative algebra $\mathfrak{A} \dagger$.

The result of our theorem is true for algebras of order one since a Jordan algebra of order one is associative and is solvable only when it is a zero algebra, $\mathfrak{A}=\mathfrak{A}^{*}$ is a zero algebra. Assume our theorem true for Jordan algebras $\mathfrak{B}$ of lower order than $\mathfrak{A}$. When $\mathfrak{A}$ is solvable $\mathfrak{B}=\mathfrak{A} \cdot 2$ is a solvable proper subalgebra of $\mathfrak{A}$. Then $\mathfrak{B}^{*}$ is nilpotent and is contained in the radical $\mathfrak{E}$ of $\mathfrak{U}^{*}$ by Lemma 7. We continue our proof by deriving

LEMMA 8. Any associative product of $s \geqq 2$ factors in $\mathfrak{A}$ with at least two factors equal is in $\mathfrak{S}$.

For such a product $p$ has a factor $d=c c_{1} \cdots c_{r} c$. If $d=c^{2}$ then $d$ is in $\mathfrak{B}$, $d$ is in the radical $\mathfrak{S}, p$ must be in $\mathfrak{S}$. Assume that all products with a factor $g g_{1} \cdots g_{r-1} g$ are in $\mathfrak{S}$ and let $p$ have a factor $d=c c_{1} \cdots c_{r} c$. Then $d=\left(c c_{1}+c_{1} c-c_{1} c\right) c_{2} \cdots c_{r} c=b c_{2} \cdots c_{r} c-c_{1} c c_{2} \cdots c_{r} c$ where $b$ is in $\mathfrak{B}$. Then 
$b c_{2} \cdots c_{r} c$ is in $\mathfrak{S}$, so is $c c_{2} \cdots c_{r} c, d$ is in $\mathfrak{S}, p$ is in $\mathfrak{S}$. This completes the inductive proof of our lemma.

Every element of $\mathfrak{A}^{*}$ is a finite sum $g=p_{1}+\cdots+p_{t}$ where each $p_{i}$ is a product of factors in $\mathfrak{A}$. Then $g^{t+1}$ is a sum of products each of $t+1$ factors $p_{i}$, at least two factors are equal, $g^{t+1}$ is nilpotent, $g$ is nilpotent. Hence all elements of $\mathfrak{I}^{*}$ are nilpotent and $\mathfrak{A}^{*}$ is a nilpotent algebra. The result that $\mathfrak{A}^{*}$ is the radical of $\mathfrak{A} \dagger$ is trivial.

We now see that if $\mathfrak{2}^{*}$ has index $k$ every associative product of $k$ elements of $\mathfrak{Q}^{*}$ is zero. But all Jordan products of $k$ elements of $\mathfrak{A}$ are sums of products of $k$ elements of $\mathfrak{A}^{*}$ and are zero, $\mathfrak{A}$ is strongly nilpotent. This completes the proof of Theorem 8 .

3. Algebras whose elements are nilpotent. We have already used the associative algebra theorem stating that if all the elements of our associative algebra are nilpotent the algebra is nilpotent. The analogous result for Lie algebras is due to Engel( $\left.{ }^{11}\right)$ and also holds for Jordan algebras. We state the result as

THEOREM 9. Let every element of a Jordan algebra $\mathfrak{A}$ be nilpotent. Then $\mathfrak{A}$ is solvable.

We shall give an inductive proof of this result and note first that the result is true for algebras of order one as well as for algebras (necessarily of order one) of linear transformations on a one-dimensional linear space. Let us then suppose that our theorem is true for any algebra $\mathfrak{B}$ whose order is less than $\mathfrak{A}$ or which is a Jordan algebra of linear transformations on a linear space of dimension less than the degree $n$ of the total matric algebra $\mathfrak{M}$ in which $\mathfrak{A}$ is imbedded. Each element of $\mathfrak{A}$ generates a solvable subalgebra of $\mathfrak{A}$ which is actually an associative nilpotent subalgebra. Let us then assume that $\mathfrak{B}$ is a proper subalgebra of $\mathfrak{A}$ which is maximal, that is, $\mathfrak{B}$ is not a proper Jordan subalgebra of any proper subalgebra of $\mathfrak{A}$. Our hypothesis implies that $\mathfrak{B}$ is solvable and that $\mathfrak{B}^{*}$ is nilpotent. By Lemma 1 there exists an idempotent linear transformation $e$ of rank $r<n$ such that $e b=b$ for every $b$ of $\mathfrak{B}$.

We may write every $a=a e+a f$ where $e$ and $f$ are pairwise orthogonal idempotents whose sum is the identity transformation. Then $e(a e)=(e a) e=c e$ and every $a e$ is a linear transformation on an $r$-dimensional linear space. If $e a=a$ for every $a$ and $c$ is in $\mathfrak{A}$ then $2 a \cdot c=2(a e+a f) \cdot(c e+c f)=2 a e \cdot c e+(a \cdot c) f$ where $(a e \cdot c e) e=a e \cdot c e$. It follows that the transformations $a e$ form a Jordan algebra and that the mapping $a \rightarrow a e$ is a homomorphism. If this mapping is an isomorphism the algebra $\mathfrak{A}$ can be imbedded in a total matric algebra of degree $r<n$ and, by the hypothesis of our induction, $\mathfrak{A}$ is solvable. Otherwise

(11) See the paper referred to in footnote 10 for a proof of Engel's theorem which does not seem applicable to the case of Jordan algebras. Our proof is an analogue of Engel's proof as given in H. Weyl's Darstellung kontinuierlicher halb-einfacher Gruppen II, Math. Zeit. vol. 24 (1925) pp. 328-376. 
the set $\mathfrak{C}$ of all elements $c$ of $\mathfrak{A}$ such that $c e=0$ is a nonzero ideal of $\mathfrak{A}$ and is solvable. The elements of $\mathfrak{A}-\mathfrak{C}$ are nilpotent, $\mathfrak{A}-\mathfrak{C}$ has lower order than $\mathfrak{A}$ and is solvable by our hypothesis, $\mathfrak{A}$ is solvable by Lemma 3.

There remains the case where $\mathfrak{A}$ contains an element $x \neq e x$. Then the set of all elements $c=e c$ of $\mathfrak{A}$ contains $\mathfrak{B}$ and is a proper subset of $\mathfrak{A}$. But $2 c_{1} \cdot c_{2}=c_{1} c_{2}+c_{2} c_{1}=\left(e c_{1}\right) c_{2}+\left(e c_{2}\right) c_{1}=2 e\left(c_{1} \cdot c_{2}\right)$. Hence this subset is a subalgebra of $\mathfrak{A}$, and our hypothesis that $\mathfrak{B}$ is a maximal subalgebra implies that $\mathfrak{B}$ consists of all quantities $a=e a$ of $\mathfrak{A}$.

We now let $x$ be an element of $\mathfrak{A}$ such that $x \neq e x$. Then either every $x \cdot b$ is in $\mathfrak{B}$ for $b$ in $\mathfrak{B}$ or there exists an $x_{1}=x \cdot b_{1} \neq e x_{1}$ for a $b_{1}$ in $\mathfrak{B}$. Similarly either every $x_{1} \cdot b$ is in $B$ or some $x_{2}=x_{1} \cdot b_{2} \neq e x_{2}$. We thus have a process of constructing a sequence of elements $x_{1}, x_{2}, \cdots, x_{t}$ such that every $x_{i+1}=x_{i} \cdot b_{i+1}$ for $b_{i+1}$ in $\mathfrak{B}$ and each $x_{i} \neq e x_{i}$. The construction terminates when we arrive at an $x_{t}$ such that $x_{t} \cdot b$ is in $\mathfrak{B}$ for every $b$ of $\mathfrak{B}$. But $t<2 k$ where $k$ is the index of $B^{*}$ since an $x_{2 k}$ is a sum of associative products of the form $c_{1} \cdots c_{8} x c_{8+1} \cdots c_{2 k}$ with the $c_{i}$ in $\mathfrak{B}^{*}$ and either $s \geqq k$ or $2 k-s \geqq k, x_{2 k}=0$.

We have now proved the existence of a quantity $y \neq e y$ in $\mathfrak{A}$ such that $y \cdot b=e(y \cdot b)$ for every $b$ of $\mathfrak{B}$. We now propose to prove that $y^{t} \cdot b=e\left(y^{t} \cdot b\right)$ for every $t$ and shall therefore assume the relation for $t$ fixed and compute $2 y^{t+1} \cdot b=y^{t+1} b+b y^{t+1}=y\left(y^{t} b+b y^{t}\right)-y b y^{t}+\left(b y^{t}+y^{t} b\right) y-\left(y^{t} b\right) y$ $=y\left(y^{t} b+b y^{t}\right)+\left(b y^{t}+y^{t} b\right) y-(y b+b y) y^{t}-\left(y^{t} b+b y^{t}\right) y+2 b y^{t+1}$ $=4 y \cdot\left(y^{t} \cdot b\right)-2(y \cdot b) y^{t}-2\left(y^{t} \cdot b\right) y+2 b y^{t+1}$. Now $y^{t} \cdot b$ is in $B$ and also $4 y \cdot\left(y^{t} \cdot b\right)$ is in $\mathfrak{B}$. The quantity $y \cdot b=e(y \cdot b), e\left(y^{t} \cdot b\right)=y^{t} \cdot b, e b=b$ so that $e\left(2 y^{t+1} \cdot b\right)$ $=2 y^{t+1} \cdot b$ and $y^{t+1} \cdot b$ is in $\mathfrak{B}$ for every $b$. We have proved that if $\mathfrak{Y}$ is the set of all polynomials $\alpha_{1} y+\alpha_{2} y^{2}+\cdots+\alpha_{t} y^{t}$ then $\mathfrak{Y} \cdot \mathfrak{B} \leqq \mathfrak{B}$. Since $y$ is nilpotent $\mathfrak{V}$ is a solvable algebra, the space $\mathfrak{Y}+\mathfrak{B}$ has the property that $(\mathfrak{Y}+\mathfrak{B}) \cdot(\mathfrak{Y}+\mathfrak{B})$ $=\mathfrak{Y} \cdot{ }^{2}+\mathfrak{Y} \cdot \mathfrak{B}+\mathfrak{B} \cdot{ }^{2} \leqq \mathfrak{Y} \cdot{ }^{2}+\mathfrak{B}$. Hence $\mathfrak{Y}+\mathfrak{B}$ is a subalgebra of $\mathfrak{A}$ properly containing $\mathfrak{B}, \mathfrak{V}+\mathfrak{B}=\mathfrak{A}, \mathfrak{B}$ is a solvable ideal of $\mathfrak{A}, \mathfrak{A}$ is solvable. This completes our proof.

\section{Corollary. A nilpotent Jordan algebra is solvable.}

For if $\mathfrak{A}$ is nilpotent the special products with all factors equal are powers of elements of $\mathfrak{A}$, all elements of $\mathfrak{A}$ are nilpotent, $\mathfrak{A}$ is solvable.

We have now shown that the concepts of solvability, nilpotency, and strong nilpotency are equivalent in the case of Jordan algebras.

4. The radical of a Jordan algebra. We shall be able to show that if $\mathfrak{N}$ is the maximal solvable ideal of a Jordan algebra then $\mathfrak{A}-\mathfrak{R}$ is a Jordan algebra and is a direct sum of simple Jordan algebras. We shall therefore call $\mathfrak{N}$ the radical of $\mathfrak{A}$ and shall say that $\mathfrak{A}$ is semisimple when $\mathfrak{R}=0$. We now prove

THEOREM 10. The radical $\mathfrak{R}$ of a Jordan algebra $\mathfrak{A}$ is the intersection of $\mathfrak{A}$ with the radical $\mathfrak{S}$ of $\mathfrak{P}^{*}$.

For, by Theorem $8, \mathfrak{N}^{*}$ is nilpotent. By Lemma $7, \mathfrak{N}^{*} \leqq \mathfrak{S}$ and hence $\mathfrak{N}$ 
is contained in the intersection $\mathfrak{D}$ of $\mathfrak{T}$ and $\mathfrak{A}$. Conversely let $d$ be in $\mathfrak{D}$. Then $d a+a d$ is in $\mathfrak{A}$ as well as in $\mathfrak{S}, d a+a d$ is in $\mathfrak{D}$ for every $a$ of $\mathfrak{A}$. It follows that $\mathfrak{D}$ is an ideal of $\mathfrak{A}$. Evidently $\mathfrak{D}$ is strongly nilpotent, $\mathfrak{D} \leqq \mathfrak{N}, \mathfrak{D}=\mathfrak{N}$.

We now propose to show that $\mathfrak{A}-\mathfrak{N}$ is a Jordan algebra. We map $\mathfrak{A}^{*}$ on the associative algebra $\mathfrak{2}^{*}-\mathfrak{S}$ by means of the mapping $a \rightarrow[a]$. Then $a b+b a \rightarrow[a b+b a]=[a][b]+[b][a]$ and so the set $\mathfrak{A}_{0}$ of all classes $[a]$ defined for $a$ in $\mathfrak{A}$ is a linear subspace of $\mathfrak{A}-\mathfrak{N}$ which is a Jordan algebra with respect to the product operation $[a] \cdot[b]=[a \cdot b]$. It follows that the mapping $a \rightarrow[a]$ is a homomorphism of $\mathfrak{A}$ on $\mathfrak{A}_{0}$. By Theorem 10 and the fact that $a \rightarrow 0$ if and only if $a$ is in $\mathfrak{E}$ we see that $a \rightarrow 0$ for $a$ in $\mathfrak{A}$ if and only if $a$ is in $\mathfrak{N}$. Hence $\mathfrak{A}-\mathfrak{N}$ is isomorphic to the Jordan algebra $\mathfrak{A}_{0}$.

5. Decomposition relative to an idempotent. Theorem 9 implies that a nonsolvable Jordan algebra $\mathfrak{A}$ contains a nonnilpotent quantity $a$. The algebra of all polynomials in $a$ is an associative algebra containing a nonnilpotent element and so contains an idempotent $e$ which is now also in $\mathfrak{A}$. It will be convenient to use matrix notations and so we can use a matrix representation of our linear transformations such that

$$
e=\left(\begin{array}{ll}
I & 0 \\
0 & 0
\end{array}\right)
$$

where $I$ is an $r$-rowed identity matrix. Let us assume that $r<n$.

Every $a$ of $\mathfrak{A}$ has the form

$$
a=\left(\begin{array}{cc}
A_{1} & A_{2} \\
A_{3} & A_{4}
\end{array}\right)
$$

where $A_{1}$ is an $r$-rowed square matrix, $A_{2}$ is an $r$ by $n-r$ matrix, $A_{3}$ is an $n-r$ by $r$ matrix, $A_{4}$ is an $(n-r)$-rowed square matrix. Then

$$
2 e \cdot a=\left(\begin{array}{cc}
A_{1} & A_{2} \\
0 & 0
\end{array}\right)+\left(\begin{array}{cc}
A_{1} & 0 \\
A_{3} & 0
\end{array}\right)
$$

and so

$$
e \cdot a=\left(\begin{array}{cc}
A_{1} & A_{2} / 2 \\
A_{3} / 2 & 0
\end{array}\right)
$$

Then

$$
e \cdot(e \cdot a)=\left(\begin{array}{cc}
A_{1} & A_{2} / 4 \\
A_{3} / 4 & 0
\end{array}\right) .
$$

It follows that $\mathfrak{A}$ contains

$$
b_{\odot}=4 e \cdot a-4 e \cdot(e \cdot a)=\left(\begin{array}{cc}
0 & A_{2} \\
A_{8} & 0
\end{array}\right)
$$


as well as

$$
a_{e}=e \cdot a-b_{e} / 2=\left(\begin{array}{cc}
A_{1} & 0 \\
0 & 0
\end{array}\right), \quad c_{e}=a-a_{e}-b_{e}=\left(\begin{array}{cc}
0 & 0 \\
0 & A_{4}
\end{array}\right) .
$$

Note that $a_{e}$ is the associative product eae.

We have now proved that $\mathfrak{A}$ is the supplementary sum $\mathfrak{A}=\mathfrak{A}_{e}+\mathfrak{B}_{e}+\mathfrak{C}_{e}$ of three subspaces $\mathfrak{A}_{e}, \mathfrak{B}_{e}, \mathfrak{E}_{e}$ of $\mathfrak{A}$. Every $a_{e}$ of $\mathfrak{A}_{e}$ has the property $a_{e} \cdot \boldsymbol{e}=a_{e} e$ $=e a_{e}=a_{e}$. Every $b_{e}$ of $\mathfrak{B}_{e}$ has the property $\boldsymbol{b} \cdot e=b / 2$ and every $c$ of $\mathfrak{E}_{e}$ has the property $c \cdot e=c e=e c=0$. Then a quantity $c$ of $\mathfrak{A}$ is orthogonal to an idempotent $e$ in the sense that $c \cdot e=0$, if and only if $c e=e c=0$. Note that $\mathfrak{A}_{e}$ is the associative product $e \mathfrak{A} e$.

The subspaces $\mathfrak{A}_{e}$ and $\mathfrak{E}_{e}$ are clearly Jordan subalgebras of $\mathfrak{A}$. However

$$
2\left(\begin{array}{cc}
0 & B_{2} \\
B_{3} & 0
\end{array}\right) \cdot\left(\begin{array}{cc}
0 & D_{2} \\
D_{3} & 0
\end{array}\right)=\left(\begin{array}{cc}
B_{2} D_{3}+D_{2} B_{3} & 0 \\
0 & B_{3} D_{2}+D_{3} B_{2}
\end{array}\right)
$$

and we have the first of the relations

$$
\mathfrak{B}_{e}^{\cdot 2} \leqq \mathfrak{A}_{\epsilon}+\mathfrak{C}_{e}, \quad \mathfrak{A}_{\epsilon} \cdot \mathfrak{B}_{\epsilon} \leqq \mathfrak{B}_{e}, \quad \mathfrak{C}_{\epsilon} \cdot \mathfrak{B}_{\epsilon} \leqq \mathfrak{B}_{\epsilon} .
$$

The last two relations follow from

$$
\begin{aligned}
& 2\left(\begin{array}{cc}
0 & B_{2} \\
B_{3} & 0
\end{array}\right) \cdot\left(\begin{array}{cc}
A_{1} & 0 \\
0 & 0
\end{array}\right)=\left(\begin{array}{cc}
0 & A_{1} B_{2} \\
B_{3} A_{1} & 0
\end{array}\right), \\
& 2\left(\begin{array}{cc}
0 & B_{2} \\
B_{3} & 0
\end{array}\right) \cdot\left(\begin{array}{cc}
0 & 0 \\
0 & A_{4}
\end{array}\right)=\left(\begin{array}{cc}
0 & B_{2} A_{4} \\
A_{4} B_{3} & 0
\end{array}\right) .
\end{aligned}
$$

6. The assumption of nonmodularity. If $A=\left(a_{i j}\right)$ is any $n$-rowed square matrix the trace of $A$ is the sum

$$
\tau(A)=a_{11}+\cdots+a_{n n}
$$

of the diagonal elements of $A$. This function is a linear function and we also have the well known

Lemma 9. Let $B$ be an $m$ by $n$ matrix and $C$ be an $n$ by $m$ matrix. Then $\tau(B C)=\tau(C B)$.

For let $B=\left(b_{i j}\right)$ and $C=\left(c_{j i}\right)$ where $i=1, \cdots, m$ and $j=1, \cdots, n$. The diagonal elements of $A=B C$ are $a_{i i}=\sum_{j=1}^{n} b_{i j} c_{j i}$ and $\tau(B C)=\sum_{i=1}^{m}\left(\sum_{j=1}^{n} b_{i j} c_{j i}\right)$. Similarly $\tau(C B)=\sum_{j=1}^{n} \sum_{i=1}^{m} c_{j i} b_{i j}$. These two finite sums of commutative products are evidently equal.

We shall think of the elements of our Jordan algebras as being $n$-rowed square matrices rather than linear transformations and note that if the characteristic of our base field $\mathfrak{F}$ is greater than $n$ then a matrix $A$ is nilpotent if and only if $\tau(A)=\tau\left(A^{2}\right)=\cdots=\tau\left(A^{n}\right)=0$. This result is a well known con- 
sequence of the Newton identities which relate the coefficients of the characteristic equation of the general matrix $A$ and the traces $\tau\left(A^{i}\right)$. In order to guarantee that this property holds we shall assume henceforth that $\mathfrak{F}$ is a nonmodular field. Note that in showing $A$ nilpotent it is sufficient to show that $\tau\left(A^{k}\right)=0$ for every $k \geqq r$ and $r$ any positive integer. For then $A^{r}$ is nilpotent and so is $A$.

7. The trace criterion for the existence of a nonzero radical. We shall establish first the following

Lemma 10. Let $a, b, c$ be in $a$ Jordan algebra and $d=a \cdot(b \cdot c)-(a \cdot b) \cdot c$. Then $\tau(d)=0$.

For $4 a \cdot(b \cdot c)=a(b c+c b)+(b c+c b) a, 4(a \cdot b) \cdot c=(a b+b a) c+c(a b+b a)$ so that $4 d=a c b+b c a-b a c-c a b=(a c) b-b(a c)+b(c a)-(c a) b$. But by Lemma 9 we see that $\tau[(a c) b-b(a c)]=\tau[b(c a)-(c a) b]=0$.

We may now derive the trace criterion as expressed in

THeOREM 11. Let $\mathfrak{A}$ be a Jordan algebra over a nonmodular field $\mathfrak{F}$. Then the radical $\mathfrak{N}$ of $\mathfrak{A}$ is the set of all elements $q$ of $\mathfrak{A}$ such that $\tau(a \cdot q)=0$ for every a of $\mathfrak{A}$.

For if $q$ is in $\mathfrak{R}$ then $a \cdot q$ is in $\mathfrak{N}$ and $q$ and $a \cdot q$ are nilpotent linear transformations, $\tau(a \cdot q)=0$. Hence $\mathfrak{R}$ is contained in the set $\mathfrak{R}$ of all $q$ 's of $\mathfrak{A}$ such that $\tau(a \cdot q)=0$ for every $a$. Conversely let $q$ and $r$ be in $\mathfrak{R}$ and $\lambda$ and $\mu$ be in $\mathfrak{F}$. Then if $s=\lambda q+\mu r$ we have $\tau(a \cdot s)=\lambda \tau(a \cdot q)+\mu \tau(a \cdot r)=0$. Hence $\mathbb{R}$ is a linear subspace of $\mathfrak{A}$. We now let $b$ be in $\mathfrak{A}$ and $q$ be in $\mathfrak{R}$ and define $r=b \cdot q$. Then $a \cdot r=(a \cdot b) \cdot q+d$ where $\tau(d)=0$ by Lemma 10 . Since $g=a \cdot b$ is in $\mathfrak{A}$ we see that $\tau(g \cdot q)=0, \tau(a \cdot r)=0, \mathbb{R}$ is an ideal of $\mathfrak{A}$. Then $q^{k-1} \cdot q=q^{k}$ and $\tau\left(q^{k}\right)=0$ for every $q$ of $\mathfrak{R}$, and every $k \geqq 2$, all elements of $\mathfrak{R}$ are nilpotent. By Theorem 9 the ideal $\mathfrak{R}$ is solvable and $\mathfrak{R}=\mathfrak{R}$ by Theorem 3 . Hence $\mathfrak{R}=\mathfrak{N}$.

Theorem 11 is used to show that if $q$ is a nonzero quantity such that $\tau(a \cdot q)=0$ for every $a$ then the radical of $\mathfrak{A}$ is not zero. The result may also be stated as

THEOREM 12. Let $\mathfrak{A}$ be a semisimple Jordan algebra over a nonmodular field $\mathfrak{F}$ and $q$ be $a$ quantity of $A$ such that $\tau(a \cdot q)=0$ for every $a$ of $\mathfrak{A}$. Then $q=0$.

We also have

THEOREM 13. Let $\mathfrak{A}$ be a Jordan algebra over a nonmodular field $\mathfrak{F}$ and $\Re$ be a scalar extension of $\mathfrak{F}$. Then if $\mathfrak{N}$ is the radical of $\mathfrak{A}, \mathfrak{N}_{\Omega}$ is the radical of $\mathfrak{A}_{\Omega}$. Thus $\mathfrak{A}$ is semisimple if and only if every scalar extension $\mathfrak{A}_{\mathfrak{R}}$ of $\mathfrak{A}$ is semisimple.

For every element of $\mathfrak{A}_{\Re}$ can be written in the form $y=\xi_{1} y_{1}+\cdots+\xi_{r} y_{r}$ where $\xi_{1}, \cdots, \xi_{r}$ are elements of $\Re$ linearly independent in $\mathfrak{F}$, and $y_{1}, \cdots, y_{r}$ are in $\mathfrak{A}$. If $y$ is in the radical $\mathfrak{R}_{0}$ of $\mathfrak{A}_{\Omega}$ then $\tau(a \cdot y)=\xi_{1} \tau\left(a \cdot y_{1}\right)+\cdots+\xi_{r} \tau\left(a \cdot y_{r}\right)$ 
$=0$ for every $a$ of $\mathfrak{A}, \tau\left(a \cdot y_{1}\right)=\cdots=\tau\left(a \cdot y_{r}\right)=0$. Hence $y_{1}, \cdots, y_{r}$ are in $\mathfrak{R}$, $y$ is in $\mathfrak{N}_{\Omega}, \mathfrak{N}_{0} \leqq \mathfrak{N}_{\Omega}$. Conversely if $y$ is in $\mathfrak{N}_{\Omega}$ and $a=\eta_{1} a_{1}+\cdots+\eta_{s} a_{s}$ is in $\mathfrak{A}_{\Omega}$ for $\eta_{i}$ in $\Re$ and $a_{i}$ in $\mathfrak{A}$ then $\tau(a \cdot y)=\sum \eta_{i} \xi_{j} \tau\left(a_{i} \cdot y_{j}\right)=0, \mathfrak{R}_{\Re} \leqq \mathfrak{N}_{0}, \mathfrak{N}_{\Re}=\mathfrak{N}_{0}$.

8. The radical of $\mathfrak{A}_{e}$ and $\mathfrak{E}_{e}$. Let $e$ be an idempotent of a nonsolvable Jordan algebra $\mathfrak{A}$ and decompose $\mathfrak{A}$ relative to $e$ as in

$$
\mathfrak{A}=\mathfrak{A}_{\bullet}+\mathfrak{B}_{\bullet}+\mathfrak{E}_{\Theta},
$$

where $e$ is the unity element of $\mathfrak{A}_{e}=e \mathfrak{A} e$. Then, as in the case of associative algebras, we may prove

THEOREM 14. Let $\mathfrak{R}$ be the radical of $\mathfrak{A}$. Then the intersection of $\mathfrak{R}$ and $\mathfrak{A}$. is the radical $\mathfrak{N}_{e}$ of $\mathfrak{A}_{e}$, the intersection of $\mathfrak{N}$ and $\mathfrak{E}_{\mathrm{e}}$ is the radical of $\mathfrak{E}_{\mathrm{e}}$.

For the radical $\mathfrak{R}_{e}$ of $\mathfrak{A}_{e}$ is the set of all elements $y$ in $\mathfrak{A}_{e}$ such that $\tau\left(a_{e} \cdot y\right)=0$ for every $a_{e}$ of $\mathfrak{A}_{e}$. Then every $a=a_{e}+b_{e}+c_{e}$ and $\tau(a \cdot y)=\tau\left(a_{e} \cdot y\right)$ $+\tau\left(b_{e} \cdot y\right)+\tau\left(c_{e} \cdot y\right)$. However $c_{e} \cdot y=0$ since $\mathfrak{A}_{e}$ and $\mathfrak{S}_{e}$ are orthogonal and $y$ is in $\mathfrak{A}_{e}$. Also, by (12), $b_{e} \cdot y$ is in $\mathfrak{B}_{e}$ and it is obvious that $\tau(b)=0$ for every $b$ of $\mathfrak{B}_{\text {e. Thus }} \tau(a \cdot y)=0$ for every $a, y$ is in $\mathfrak{N}$ and in $\mathfrak{A}_{e}$. It follows that $\mathfrak{R}_{e}$ is contained in the intersection of $\mathfrak{N}$ and $\mathfrak{A}_{e}$. The converse is trivial. The radical of $\mathfrak{E}_{e}$ is the set of all quantities $z$ in $\mathfrak{E}_{e}$ such that $\tau(c \cdot z)=0$ for every $c$ of $\mathfrak{E}_{e}$. and $a \cdot z=a_{e} \cdot z+b_{e} \cdot z=0+b_{1 e}+c_{e} \cdot z, \tau(a \cdot z)=0$. As before the radical of $\mathfrak{G}_{e}$ is contained in the desired intersection and the trivial converse completes our proof.

If $y$ is in $\mathscr{B}_{e}$ and $a=a_{e}+b_{e}+c_{e}$ as in the decomposition (14) then $a \cdot y=a_{e} \cdot y+c_{e} \cdot y+b_{e} \cdot y$ and, by (12), $a_{e} \cdot y+c_{e} \cdot y$ is in $\mathfrak{B}_{e}, \tau(a \cdot y) \stackrel{\prime}{=} \tau\left(b_{e} \cdot y\right)$. As a conclusion we may state

THEOREM 15. An element $y$ of $\mathfrak{B}_{e}$ is in the radical of $\mathfrak{A}$ if and only if $\tau(b \cdot y)$ $=0$ for every $b$ of $\mathfrak{B}_{\mathrm{e}}$.

9. Principal idempotents. An idempotent $c$ of a Jordan algebı a $\mathfrak{A}$ is called a principal idempotent if there exists no idempotent $d$ in $\mathfrak{A}$ such that $d \cdot c=0$. Then $\mathfrak{E}_{e}$ must be solvable, $\mathfrak{E}_{e} \leqq \mathfrak{N}$, the radical of $\mathfrak{A}$. Indeed, as for associative algebras, we may prove

THEOREM 16. Let e be a principal idempotent of $\mathfrak{A}$. Then $\mathfrak{B}_{\bullet}+\mathfrak{C}_{\mathrm{e}}$ is contained in the radical $\mathfrak{R}$ of $A$.

For $\mathfrak{S}_{e} \leqq \mathfrak{N}$ and, by Theorem 15 , it is sufficient to prove that if $y$ and $b$ are in $\mathfrak{B}_{\text {e }}$ then $\tau(b \cdot y)=0$. Write

and see that

$$
y=\left(\begin{array}{cc}
0 & Y_{2} \\
Y_{3} & 0
\end{array}\right), \quad b=\left(\begin{array}{cc}
0 & B_{2} \\
B_{3} & 0
\end{array}\right)
$$

$$
2 y \cdot b=y b+b y=\left(\begin{array}{cc}
Y_{2} B_{3}+B_{2} Y_{3} & 0 \\
0 & Y_{3} B_{2}+B_{3} Y_{2}
\end{array}\right) .
$$


Then

$$
\left(\begin{array}{cc}
0 & 0 \\
0 & Y_{3} B_{2}+B_{3} Y_{2}
\end{array}\right)
$$

is in $\mathfrak{C}_{e}$ and in $\Re, \tau\left(Y_{3} B_{2}+B_{3} Y_{2}\right)=\tau\left(Y_{3} B_{2}\right)+\tau\left(B_{3} Y_{2}\right)=0$. But by Lemma 9 we have $\tau\left(Y_{2} B_{3}+B_{2} Y_{3}\right)=\tau\left(Y_{2} B_{3}\right)+\tau\left(B_{2} Y_{3}\right)=\tau\left(B_{3} Y_{2}\right)+\tau\left(Y_{3} B_{2}\right)=0, \tau(2 y \cdot b)$ $=2 \tau(y \cdot b)=0, \tau(y \cdot b)=0$.

If $u$ is any nonprincipal idempotent of $\mathfrak{A}$ the algebra $\mathfrak{E}_{u}$ is not solvable and contains an idempotent $v$ such that $u+v$ has rank equal to the rank of $u$ plus the rank of $v$. Hence an idempotent of maximum rank in $\mathfrak{A}$ is necessarily principal. We have proved

THEOREM 17. Every nonsolvable Jordan algebra contains a principal idempotent.

10. The structure theorem for semisimple algebras. We first prove

THEOREM 18. Let $\mathfrak{A}$ be semisimple. Then $\mathfrak{A}$ contains a unity element e such that $e a=a e=e \cdot a=a$ for every $a$ of $\mathfrak{A}, \mathfrak{A}=e \mathfrak{A} e$.

For $\mathfrak{C}_{e}+\mathfrak{B}_{e}=0, \mathfrak{A}=\mathfrak{A}_{e}$ and it has already been observed that $e a=a e=a \cdot e$ $=a$ for every $a$ of $\mathfrak{A}_{e}$.

By Theorem 14 if $e$ is any idempotent of a semisimple algebra $\mathfrak{A}$ the algebra $\mathfrak{A}_{\bullet}$ is semisimple and $e$ is its unity element. Also $\mathfrak{E}_{6}$ is semisimple. We now prove

THEOREM 19. Every ideal $\mathfrak{D}$ of a semisimple Jordan algebra $\mathfrak{A}$ is semisimple, $\mathfrak{D}=\mathfrak{A}_{e}$ for an idempotent e such that $\mathfrak{A}=\mathfrak{D} \oplus \mathfrak{C}_{e}$.

For no ideal $\mathfrak{D}$ of $\mathfrak{A}$ can be solvable, $\mathfrak{D}$ contains a principal idempotent $e$. We decompose $\mathfrak{A}$ relative to $e$ and write $\mathfrak{A}=\mathfrak{A}_{e}+\mathfrak{B}_{e}+\mathfrak{C}_{\bullet}$ and similarly write $\mathfrak{D}=\mathfrak{A}_{1 e}+\mathfrak{B}_{1 e}+\mathfrak{C}_{1 e}$. But $\mathfrak{D}$ is an ideal containing $e$ and so every $b_{e}=4 e \cdot a$ $-e \cdot(e \cdot a)$ is in $\mathfrak{D}$, every $a_{e}=e \cdot a-b_{e} / 2$ is in $\mathfrak{D}$. It follows that $\mathfrak{D}=\mathfrak{A}_{e}+\mathfrak{B}_{e}+\mathfrak{C}_{1_{e}}$ where $\mathfrak{C}_{1 e}$ is the intersection of $\mathfrak{D}$ and $\mathfrak{C}_{e}$. By Theorem $16, \mathfrak{B}_{e}+\mathfrak{C}_{1 e}$ is in the radical of $\mathfrak{D}$ and $\tau(b \cdot y)=0$ for every $b$ and $y$ of $\mathscr{B}_{e}$. Then $\mathscr{B}_{\bullet}$ is in the radical of $\mathfrak{A}$ by Theorem 15 . Hence $\mathfrak{B}_{e}=0$. Now $\mathfrak{E}_{1 e} \cdot \mathfrak{C}_{e} \leqq \mathfrak{D}$ since $\mathfrak{D}$ is an ideal, $\mathfrak{E}_{1 e} \cdot \mathfrak{G}_{e} \leqq \mathfrak{G}_{e}$. Hence $\mathfrak{G}_{1 e}$ is a solvable ideal of $\mathfrak{C}_{e}$, whereas $\mathfrak{C}_{e}$ is semisimple. Then $\mathfrak{E}_{1 e}=0$ and $\mathfrak{D}=\mathfrak{A}_{e}, \mathfrak{A}=\mathfrak{A}_{e} \oplus \mathfrak{E}_{e}$ as desired.

We now have our principal result

THEOREM 20. Every semisimple Jordan algebra over a nonmodular field is a direct sum of simple algebras.

For if $\mathfrak{A}$ is semisimple but not simple it contains an ideal $\mathfrak{B}$ and $\mathfrak{A}=\mathfrak{B} \oplus \mathfrak{C}$ where $\mathfrak{B}$ and $\mathfrak{C}$ are semisimple. If either $\mathfrak{B}$ or $\mathfrak{C}$ is not simple we decompose again and ultimately obtain a decomposition $\mathfrak{A}=\mathfrak{B}_{1}+\cdots+\mathfrak{B}_{r}$ where the 
components are semisimple algebras containing no ideals, and hence no component is a zero algebra, each component is simple.

It should be noted that Theorem 19 does not follow from Theorem 4 in which the concept of semisimplicity was that of the property proved in Theorem 20 .

\section{Chapter III. Simple Jordan algebras}

1. The center of a Jordan algebra. The center $\mathfrak{S}$ of a Jordan algebra $\mathfrak{A}$ is defined to be the set of all quantities $c$ in $\mathfrak{A}$ such that the associative products $a c$ and $c a$ are equal for every $a$ of $\mathfrak{A}$. Then

$$
c \cdot a=(c a+a c) / 2=c a
$$

for every $a$ of $\mathfrak{A}$ and $c$ of $\mathbb{E}$ and it should be clear that $\mathbb{E}$ is zero or a subalgebra of $\mathfrak{A}$ which is both commutative and associative.

If $\mathfrak{A}$ is simple then the unity element of $\mathfrak{A}$ is in $\mathfrak{E}$. We may indeed prove

THEOREM 21. The center of a simple Jordan algebra is a field.

For if $\mathfrak{C}$ has a radical $\mathfrak{N}$ and $h \neq 0$ is in $\mathfrak{N}$ then $h \cdot a=h a=a h$ and $(h \cdot a)^{k}=(h a)^{k}=h^{k} a^{k}=0$ for $k$ the index of $\mathfrak{N}$. Then every $h \cdot a$ is nilpotent and $h$ is in the radical of $\mathfrak{A}$ contrary to our hypothesis that $\mathfrak{A}$ is simple. Hence $\mathfrak{S}$ is semisimple. If $\mathfrak{E}$ is not simple the unity element $e$ of $\mathfrak{A}$ is expressible as a sum of two pairwise orthogonal idempotents $u$ and $v$ of $\mathbb{E}$ and $a \cdot e=a=a(u+v)$ $=a \cdot u+a \cdot v, \mathfrak{A}$ is readily seen to be the direct sum of $\mathfrak{A} u$ and $\mathfrak{A} v$. It follows that the commutative associative algebra $\sqrt{ }$ is simple and thus that $\mathbb{} \subseteq$ is a field.

With every $a$ and $b$ of $\mathfrak{A}$ and $\alpha$ and $\beta$ of $\mathfrak{S}$ the algebra $\mathfrak{A}$ contains $\alpha a+\beta b$. Then $\mathfrak{A}$ may be regarded as a linear space over $\mathfrak{E}$. Moreover $\mathfrak{E}$ is a field of linear transformations commutative with all linear transforniations of $\mathfrak{A}$ and it is well known that the quantities of $\mathfrak{A}$ may then be regarded as linear transformations over $\mathfrak{C}$. Hence $\mathfrak{A}$ may be expressed as a Jordan algebra of linear transformations over $\mathfrak{C}$ and is evidently simple over $\mathfrak{E}$.

We define a Jordan algebra $\mathfrak{A}$ to be central simple if the center of $\mathfrak{A}$ is the field $e \mathfrak{F}$ of order one over $\mathfrak{F}$. Then we have shown above that every simple Jordan algebra is expressible as a central simple Jordan algebra over its center. We now prove

THEOREM 22. Every scalar extension of a central simple Jordan algebra $\mathfrak{A}$ is central simple.

For if $\mathfrak{R}$ is a scalar extension of the base field $\mathfrak{F}$ of $\mathfrak{A}$ the algebra $\mathfrak{A}_{\Omega}$ is semisimple by Theorem 13. If $\mathfrak{A}_{\Re}$ is not simple the unity element $e$ of $\mathfrak{A}$ is expressible in the form $e=u+v$ for pairwise orthogonal idempotents $u$ and $v$ in the center of $\mathfrak{A}_{\Omega}$. Then we may write $u=u_{1} \xi_{1}+\cdots+u_{r} \xi_{r}$ where $u_{1}, \cdots, u_{r}$ are in $\mathfrak{A}$ and $\xi_{1}, \cdots, \xi_{r}$ are elements of $\Re$ such that $\sum_{i=1}^{r} a_{i} \xi_{i}=0$ for $a_{i}$ in $\mathfrak{A}^{*}$ if and only if $a_{1}=\cdots=a_{r}=0$. But $u a-a u=\sum_{i=1}^{r}\left(u_{i} a-a u_{i}\right) \xi_{i}=0$, 
$u_{i} a=a u_{i}$, the $u_{i}$ are in the center of $\mathfrak{A}, u_{i}=\alpha_{i} e$ for $\alpha_{i}$ in $\mathfrak{F}, u=\left(\sum_{i=1}^{r} \alpha_{i} \xi_{i}\right) e$ is not a singular idempotent. This completes our proof.

2. Primitive idempotents. An idempotent $u$ of a Jordan algebra $\mathfrak{A}$ is said to be primitive in $\mathfrak{A}$ if $u \neq v+w$ for any pairwise orthogonal idempotents $v$ and $w$ of $\mathfrak{A}$. This can occur if and only if $u$ is the only idempotent of the algebra $\mathfrak{A}_{u}$ defined in the decomposition of $\mathfrak{A}$ relative to $u$. For if $u=v+w$ then $u v=v^{2}+w v=v=v u, v$ is in $\mathfrak{A}_{u}, w$ is in $\mathfrak{A}_{u}$ and $u \neq v$. Conversely if $\mathfrak{A}_{u}$ contains an idempotent $v \neq u$ then $u v=v u=v$ and $w=u-v$ is an idempotent and is orthogonal to $u$ by the well known considerations of associative algebra theory.

The algebra $\mathfrak{A}_{u}$ has $u$ as its unity element and we may regard $u$ as being the identity transformation for $\mathfrak{A}_{u}$. We may indeed write

$$
a=\left(\begin{array}{cc}
A_{1} & 0 \\
0 & 0
\end{array}\right), \quad u=\left(\begin{array}{cc}
I & 0 \\
0 & 0
\end{array}\right)
$$

for every $a$ of $\mathfrak{A}_{u}$ where $A_{1}$ is an $r$-rowed square matrix and $r$ is the rank of $u$, and may say that $a$ is singular or nonsingular in $\mathfrak{A}_{u}$ according as the determinant of $A_{1}$ is or is not zero. We may then prove

Lemma 11. Let $u$ be a primitive idempotent of a Jordan algebra $\mathfrak{A}$. Then the singular elements in $\mathfrak{A}_{u}$ are all nilpotent.

For if $a$ is a nonnilpotent singular element of $\mathfrak{A}_{u}$ then the polynomial algebra $\mathfrak{F}[a]$ is an associative nonnilpotent algebra contained in $\mathfrak{A}_{u}$. The elements of $\mathfrak{F}[u]$ have the form $\left(\alpha_{1} u+\alpha_{2} a+\cdots+\alpha_{r-1} a^{r-1}\right) a$ and are all singular, $\mathfrak{F}[u]$ contains an idempotent $v$ which is singular, $v \neq u$ contrary to our hypothesis that $u$ is primitive.

If $u$ is an idempotent of $\mathfrak{A}$ and is not primitive then $u=v+\psi$ where $v$ and $w$ are orthogonal idempotents. Then the rank of $u$ is the sum of the ranks of $v$ and $w$. It follows that the process of decomposing idempotents into sums of pairwise orthogonal idempotents is a finite process and, as in the associative theory, we have

LEMma 12. Every nonprimitive idempotent a of a Jordan algebra $\mathfrak{A}$ is expressible as the sum of a finite number of pairwise orthogonal idempotents $u_{i}$ of $A$ such that $u u_{i}=u_{i} u=u_{i}$.

Lemma 13. Let $u$ be a nonprincipal idempotent of $\mathfrak{A}$. Then there exists a principal idempotent $e=e_{1}+\cdots+e_{t}$ for pairwise orthogonal primitive idempotents $e_{i}$ such that $u=e_{1}+\cdots+e_{s}, s<t$.

3. Decomposition of an algebra relative to several idempotents. If $\mathfrak{A}$ is a Jordan algebra with a unity element $e$ we have seen that there exists a matrix representation of $\mathfrak{A}$ such that $e$ is the identity matrix. Let us select such a representation of $\mathfrak{A}$ by $n$-rowed square matrices and express $e$ as a sum 


$$
e=e_{1}+\cdots+e_{t}
$$

of pairwise orthogonal idempotents $e_{i}$ which are in $\mathfrak{A}$. Then every $a$ of $\mathfrak{A}$ may be partitioned as a matrix

$$
a=\left(A_{i j}\right) \quad(i, j=1, \cdots, t),
$$

where the number of rows in $A_{i j}$ is the rank of $e_{i}$, and the number of columns in $A_{i j}$ is the rank of $e_{j}$. Then

$$
e_{i} a e_{j}=\left(B_{p q}\right)
$$

where $B_{i j}=A_{i j}$ and $B_{p q}=0$ for $p \neq i$ and $q \neq j$. The matrices $e_{i} a e_{j}$ need not be in $\mathfrak{A}$. However every

$$
a_{i i}=e_{i} a e_{i}
$$

is in $\mathfrak{A}_{e_{i}}=\mathfrak{A}_{i i}=e_{i} \mathfrak{A} e_{i}=\mathfrak{A}$ and indeed an element $a$ of $\mathfrak{U}$ is in $\mathfrak{A}_{i i}$ if and only if $a=e_{i} a e_{i}$.

For every $i \neq j$ we form

$$
4 e_{j} \cdot\left(a \cdot e_{i}\right)=\left(e_{i} a+a e_{i}\right) e_{j}+e_{j}\left(e_{i} a+a e_{i}\right)=e_{i} a e_{j}+e_{j} a e_{i}=a_{i j} .
$$

Then each

$$
a_{i j}=4 e_{i} \cdot\left(a \cdot e_{j}\right)=4 e_{j} \cdot\left(a \cdot e_{i}\right)=a_{j i}
$$

is in $\mathfrak{A}$ and the set $\mathfrak{A}_{i j}=\mathfrak{A}_{j i}$ of all $a_{i j}$ is a linear subspace of $\mathfrak{A}$. It should be clear that $\mathfrak{A}$ is the supplementary sum of its $t(t+1) / 2$ subspaces $\mathfrak{A}_{i j}$ for $i, j=1, \cdots, t$ and $i \geqq j$.

With each element $a_{i i}$ of $\mathfrak{A}_{i i}$ we associate the square matrix $A_{i i}$ of (15) and with each $a_{i j}$ of $\mathfrak{A}_{i j}$ we associate the pair of rectangular matrices $\left(A_{i j}, A_{j i}\right)$. We note that each $\mathfrak{A}_{i i}$ is an algebra and the correspondence $a_{i i} \rightarrow A_{i i}$ is an isomorphism of $\mathfrak{A}_{i i}$ and the Jordan algebra of the matrices $A_{i i}$. We shall require a knowledge of the products $\mathfrak{A}_{i j} \cdot \mathfrak{A}_{p q}$ in the explicit form regarding products of resulting associated matrices and we first note that

$$
\mathfrak{A}_{i j \mathfrak{A}_{p q}}=0 \quad(i \neq p, q ; j \neq p, q) .
$$

We next compute

$$
2 a_{i i} \cdot b_{i j}=a_{i i} b_{i j}+b_{i j} a_{i i}=c_{i j}
$$

where if $a_{i i} \rightarrow A_{i i}$ and $b_{i j} \rightarrow\left(B_{i j}, B_{j i}\right)$ then, by matrix multiplication,

$$
c_{i j} \rightarrow\left(C_{i j}, C_{j i}\right), \quad C_{i j}=A_{i i} B_{i j}, \quad C_{j i}=B_{j i} A_{i i} .
$$

It follows that $\mathfrak{A}_{i i} \cdot \mathfrak{A}_{i j} \leqq \mathfrak{A}_{i j}$. Indeed if we take $a_{i i}=e_{i}$ then $2 a_{i i} \cdot b_{i j}=b_{i j}$ and so $\mathfrak{A}_{i i} \cdot \mathfrak{A}_{i j}=\mathfrak{A}_{i j}$. However we require the more explicit result of (21).

We next form

$$
2 a_{i j} \cdot b_{i j}=a_{i j} b_{i j}+b_{i j} a_{i j}=c_{i i}+c_{j j}
$$


where if $a_{i j} \rightarrow\left(A_{i j}, A_{j i}\right), b_{i j} \rightarrow\left(B_{i j}, B_{j i}\right)$ then

$$
c_{i i} \rightarrow C_{i i}=A_{i j} B_{j i}+B_{i j} A_{j i}, \quad c_{j i} \rightarrow C_{j i}=A_{j i} B_{i j}+B_{j i} A_{i j} .
$$

Evidently $\mathfrak{A}_{i j} \cdot \mathfrak{A}_{i j} \leqq \mathfrak{A}_{i i}+\mathfrak{A}_{j j}$. Finally let $i, j, k$ be distinct and form

$$
2 a_{i j} \cdot b_{j k}=a_{i j} b_{j k}+b_{j k} a_{i j}=c_{i k},
$$

where if $a_{i j} \rightarrow\left(A_{i j}, A_{j i}\right)$ and $b_{j k} \rightarrow\left(B_{j k}, B_{k j}\right)$ then

$$
c_{i k} \rightarrow\left(C_{i k}, C_{k i}\right), \quad C_{i k}=A_{j i} B_{i k}, \quad C_{k i}=B_{k i} A_{i j} .
$$

4. Decomposition relative to a set of primitive idempotents. Let the unity quantity $e$ of a simple Jordan algebra $\mathfrak{A}$ be expressed as a sum of pairwise orthogonal primitive idempotent quantities $e_{1}, \cdots, e_{t}$ of $\mathfrak{A}$. By Theorem 14 every $\mathfrak{A}_{i i}$ is semisimple and must be simple since when $\mathfrak{A}_{i i}$ is not simple it contains an idempotent distinct from its unity element $e_{i}$.

If every $A_{i j}=0$ for any $i$ and every $j \neq i$ then $\mathfrak{A}$ is the direct sum of $\mathfrak{A}_{i i}$ and $\mathfrak{S}_{e_{i}}$. This contradicts our hypothesis that $\mathfrak{A}$ is simple. Hence there exists an $\mathfrak{A}_{i j} \neq 0$ for every $i$.

If $\mathfrak{A}_{i j} \neq 0$ for $i \neq j$ there exists an $a_{i j} \neq 0$. Every $a_{i j}$ has zero trace and if every $a_{i j}{ }^{2}$ has zero trace then $\left(a_{i j}+b_{i j}\right)^{2}=a_{i j}{ }^{2}+b_{i j}{ }^{2}+2 a_{i j} \cdot b_{i j}$ has trace zero and every $a_{i j} \cdot b_{i j}$ has zero trace. By Theorem $15, \mathfrak{A}_{i j} \neq 0$ is in the radical of $\mathfrak{A}$ contrary to our hypothesis that $\mathfrak{A}$ is simple.

Now $a_{i j}{ }^{2}=b_{i i}+c_{j i}$ where $b_{i i} \rightarrow B_{i i}=A_{i j} A_{j i}$ and $c_{j i} \rightarrow C_{j j}=A_{j i} A_{i j}$. By Lemma 11 both $B_{i i}$ and $C_{j j}$ are nonsingular or nilpotent. Moreover the trace $\tau\left(B_{i i}\right)=\tau\left(C_{j i}\right)$ so that if either $B_{i i}$ or $C_{i j}$ is nilpotent then $\tau\left(a_{i j}{ }^{2}\right)=0$. It follows that there must exist some $a_{i j}$ in $A_{i j}$ such that $B_{i i}$ and $C_{j i}$ are nonsingular. However the ranks of $B_{i i}$ and $C_{j i}$ cannot exceed the lesser of the ranks of $e_{i}$ and $e_{j}$. Thus $e_{i}$ and $e_{j}$. have the same ranks, $A_{i j}$ and $A_{j i}$ must be nonsingular. We have proved

Lemma 14. Let $i \neq j$ and $\mathfrak{A}_{i j} \neq 0$. Then $e_{i}$ and $e_{j}$ have the same rank $r$ and there exists an element $a_{i j}$ in $\mathfrak{A}_{i j}$ such that $a_{i j} \rightarrow\left(A_{i j}, A_{j i}\right)$ where $A_{i j}$ and $A_{j i}$ are nonsingular r-rowed square matrices.

If $i$ and $j$ are any two distinct integers such that $\mathfrak{A}_{i j} \neq 0$ we can relabel our idempotents and so assume that $\mathfrak{A}_{12} \neq 0$. Let $\mathfrak{A}_{12}, \cdots, \mathfrak{A}_{1 k}$ be not zero and $\mathfrak{A}_{1 k+1}=\cdots=\mathfrak{A}_{1 t}=0$, a hypothesis which can be obtained by a relabeling of idempotents. Select an $a_{1 j}$ in each $\mathfrak{A}_{1 j}$ for $j=2, \cdots, k$ such that $\mathfrak{A}_{1 j}$ and $\mathfrak{A}_{j 1}$ are nonsingular and form

$$
2 a_{1 j} \cdot b_{j p}=c_{1 p}=0 \quad(p=k+1, \cdots, t)
$$

for every $b_{j p}$ of $\mathfrak{A}_{j p}$. But $c_{1 p} \rightarrow\left(C_{1 p}, C_{p 1}\right)$ where $C_{1 p}=A_{1 j} B_{j p}=0, C_{p 1}=B_{p j} A_{j 1}=0$ if and only if $B_{j p}=0, B_{p j}=0$ since $A_{1 j}$ and $A_{j 1}$ are nonsingular. Hence $A_{j p}=0$ for $j=1, \cdots, k$ and $p=k+1, \cdots, t$. It follows immediately that $\mathfrak{A}=\mathfrak{A}_{u} \oplus \mathfrak{A}_{v}$ where $u=e_{1}+\cdots+e_{k}, v=e_{k+1}, \cdots, e_{t}$. This contradicts our hypothesis 
that $\mathfrak{A}$ is simple. We have proved that every $\mathfrak{A}_{i j} \neq 0$ and thus that all of the primitive idempotents $e_{i}$ have the same rank. We may now prove

TheOREM 23. Let $\mathfrak{A}$ be a simple Jordan algebra with unity element $e=e_{1}$ $+\cdots+e_{t}$ for pairwise idempotents $e_{1}, \cdots, e_{t}$. Then the linear spaces $\mathfrak{A}_{i j}$ defined for $i \neq j$ all have the same order $s>0$, the idempotents $e_{i}$ all have the same rank $r$ and every $\mathfrak{A}_{i j}$ contains an element $e_{i j} \rightarrow\left(E_{i j}, E_{j i}\right)$ where $E_{i j}$ and $E_{j i}$ are $r$-rowed nonsingular matrices.

For we require only the proof that if $s$ is the order of $\mathfrak{A}_{12}$ then $s$ is the order of every $\mathfrak{A}_{i j}$ with $i \neq j$. It is evidently sufficient to consider the case where $t>2$ and to prove that $\mathfrak{A}_{12}, \mathfrak{A}_{13}$ and $\mathfrak{A}_{23}$ all have the same order $s$. We form $2 e_{12} \cdot b_{23}=c_{13}$ and see that $c_{13}=0$ if and only if $b_{23}=0$ since $C_{13}=E_{12} B_{23}$, $C_{31}=B_{32} E_{21}$ where $E_{12}$ and $E_{21}$ are nonsingular. Hence the order $s_{3}$ of $\mathfrak{A}_{13}$ is greater than or equal to the order $s_{2}$ of $\mathfrak{A}_{23}$. Similarly we form $2 a_{12} \cdot e_{23}=c_{13}=0$ if and only if $a_{12}=0$, and $s_{3} \geqq s$, the order of $\mathfrak{A}_{12}$. Finally we form $2 e_{12} \cdot b_{13}=c_{23}$ and $2 a_{12} \cdot e_{13}$ to see that $s_{3} \leqq s_{2}, s_{1} \leqq s_{2}$. Then $s=s_{2}=s_{3}$ as desired.

Let us note the following

CoROLlary. Let $u$ be an idempotent of a simple Jordan algebra $\mathfrak{A}$. Then $\mathfrak{A}_{u}$ is simple.

For $\mathfrak{A}_{u}$ is semisimple and is not simple only if $u=v+w, \mathfrak{A}_{u}=\mathfrak{A}_{v} \oplus \mathfrak{A}_{w}$ for pairwise orthogonal idempotents $v$ and $w$ of $\mathfrak{A}_{u}$. We may now express the unity element $e$ of $\mathfrak{A}$ in the form $e=v+w+(e-u), v=e_{1}+\cdots+e_{p}, w=e_{p+1}+\ldots$ $+e_{q}, e-u=e_{q+1}+\cdots+e_{t}$ for pairwise orthogonal primitive idempotents $e_{1}, \cdots, e_{t}$. Then $\mathfrak{A}_{i j}=0$ if $1 \leqq i \leqq p, p+1 \leqq j \leqq q$ contrary to Theorem 23 .

5. Reduced algebras. A Jordan algebra $\mathfrak{A}$ over a field $\mathfrak{F}$ will be called a reduced algebra if $\mathfrak{A}$ is a simple algebra whose unity element $e$ can be expressed as the sum $e=e_{1}+\cdots+e_{t}$ of pairwise orthogonal idempotents $e_{i}$ such that the corresponding subalgebras $e_{i} \mathfrak{A} e_{i}$ of $\mathfrak{A}$ have order one over $\mathfrak{F}$. The $e_{i}$ are then automatically primitive idempotents of $\mathfrak{A}$. They have the same rank $r$ and $e_{i} a e_{i}=\alpha_{i} e_{i}$ for every $a$ of $\mathfrak{A}$ where $\alpha_{i}$ is in $\mathfrak{F}$. If $\mathfrak{E}$ is the center of $\mathfrak{A}$ then $e_{i} \mathfrak{A} e_{i}$ contains $e_{i} \mathfrak{E}$ whose order is that of $\mathfrak{E}$. Hence a reduced algebra is central simple. We note that for every $a$ of $\mathfrak{A}$ the trace $\tau(a)=r\left(\alpha_{1}+\cdots+\alpha_{t}\right)$ so that if $\tau(a) \neq 0$ there is some $\alpha_{i} \neq 0$. We use this result in the proof of

Theorem 24. All primitive idempotents $u$ of a reduced algebra $\mathfrak{A}$ have the same rank $r$ and $u \mathfrak{A} u$ has order one for every primitive idempotent $u$ of $\mathfrak{A}$.

For $u^{k}=u$ for every $k>1$ if $u$ is idempotent and $\tau(u)=0$ implies that $\tau\left(u^{k}\right)=0, u$ is nilpotent. Hence the trace of an idempotent $u$ is not zero and $e_{i} u e_{i}=\mu e_{i} \neq 0$ for some $i$. Weform $v=u e_{i} u$ and see that $e_{i} v e_{i}=e_{i} u e_{i} e_{i} u e_{i}=\mu^{2} e_{i} \neq 0$. The rank of $v$ cannot exceed $r$ and the rank $r$ of $e_{i} v e_{i}$ cannot exceed the rank of $v$. Hence $v$ has rank $r$. But $v^{2}=u e_{i} u u e_{i} u=u\left(e_{i} u e_{i}\right) u=\mu v, \mu^{-1} v$ is an idempotent of $u \mathfrak{A} u$. If $u$ is primitive $u=\mu^{-1} v$ has rank $r$. Also $u a u=\mu^{-2} v a v$ 
$=\mu^{-2} u e_{i} u a u e_{i} u=\mu^{-2} \beta v$ where $e_{i} u a u e_{i}=\beta e_{i}$. Then $u a u=\gamma u$ for $\gamma=\mu^{-1} \beta$ in $F$ and $u \mathfrak{A} u$ has order one as desired.

As a consequence of Theorem 24 we have

THEOREM 25. A simple algebra $\mathfrak{A}$ is a reduced algebra if and only if $u \mathfrak{A} u$ has order one for every primitive idempotent $u$ of $\mathfrak{A}$. Then the number tof idempotents in the expression $e=e_{1}+\cdots+e_{t}$ of the unity quantity e of $\mathfrak{A}$ as a sum of pairwise orthogonal primitive idempotents of $\mathfrak{A}$ is unique and will be called the degree of $\mathfrak{A}$.

For by Theorem 24 if $\mathfrak{A}$ is reduced every $u \mathfrak{A} u$ has order one. The converse is an obvious consequence of our definition. The elements of $\mathfrak{A}$ are linear transformations on an $n$-dimensional space with $e$ as identity transformation, and $n=r t$ where $r$ is the rank of each $e_{i}$. But then if $e=u_{1}+\cdots+u_{q}$ for pairwise orthogonal idempotents $u_{i}$ the $u_{i}$ all have rank $r, n=r q=r t, q=t$.

6. Reduction fields. A scalar extension $\Omega$ of $\mathfrak{F}$ is called a reduction field for a central simple algebra $\mathfrak{A}$ if $\mathfrak{A}_{\mathfrak{R}}$ is a reduced algebra. We now prove

LeMmA 15. Let $\mathfrak{A}$ be a simple Jordan algebra with unity element $e$ and let $e$ be the only idempotent of $\mathfrak{A}_{\Re}$ for every scalar extension $\Omega$ of finite degree over the base field $\mathfrak{F}$. Then $\mathfrak{A}=e \mathfrak{F}$ has order one over $\mathfrak{F}$.

For suppose that $\mathfrak{A}$ has order $n>1$ over $\mathfrak{F}$. If some quantity $a$ of $\mathfrak{A}$ is a linear transformation with at least two distinct characteristic roots $\xi$ and $\eta$ we may adjoin $\xi$ to $\mathfrak{F}$ and $a-\xi e$ will be a singular nonnilpotent quantity of the resulting scalar extension $\mathfrak{A}_{\Omega}, \Re=\mathfrak{F}(\xi)$. By Lemma 11 the algebra $\Re[a-\xi e]$ will contain an idempotent $u \neq e$ and $u$ will be in $\mathfrak{A}_{\Re}$ contrary to hypothesis. Hence we may assume that every $a$ of $\mathfrak{A}_{\Re}$ has only a single characteristic root for every scalar extension $\Re$ of finite degree over $\mathfrak{F}$. Select a basis $e, u_{2}, \cdots, u_{n}$ of $\mathfrak{A}$ and extend $\mathfrak{F}$ to a field $\Re$ containing the characteristic roots $\alpha_{2}, \cdots, \alpha_{n}$ of $u_{2}, \cdots, u_{n}$ respectively. Put $v_{i}=u_{i}-\alpha_{i} e$ and have a basis $e, v_{2}, \cdots, v_{n}$ such that $v_{2}, \cdots, v_{n}$ are nilpotent. We let $\mathfrak{B}$ be the subspace of $\mathfrak{A}_{\boldsymbol{R}}$ spanned by $v_{2}, \cdots, v_{n}$ and see that if $a=\beta_{1} e+b$ where $b$ is in $\mathfrak{B}$ then $\tau(a)=\beta_{1} \tau(e)=0$ if and only if $a=b$ is in $\mathfrak{B}$. Now if $b$ is in $\mathfrak{B}$ then $b=\xi e+c$ where $\xi$ is the single characteristic root of $b$ in some finite extension of $\Omega$ and $c$ is nilpotent. Then $\tau(b)=\xi \tau(e)+\tau(c)=\xi \tau(e)=0$ and $c=b$. Hence every quantity of $\mathfrak{B}$ is nilpotent. If $b$ and $c$ are in $\mathscr{B}$ so is $b+c$ and $(b+c)^{2}=b^{2}+c^{2}+2 b \cdot c$ has zero trace, $\tau\left(b^{2}\right)=\tau\left(c^{2}\right)=0, \tau(b \cdot c)=0, b \cdot c$ is in $\mathfrak{B}$. Then $\left(\beta_{1} e+b\right) \cdot c=\beta_{1} c+b \cdot c$ is in $\mathfrak{B}$ for every $c$ of $\mathfrak{B}$ and $a=\beta_{1} e+b$ of $\mathfrak{A}, \mathfrak{B}$ is a solvable ideal of $\mathfrak{A}$ contrary to our hypothesis that $\mathfrak{A}$ is simple. It follows that our assumption $n>1$ leads to a contradiction and $n=1$ as desired.

We use the lemma above in the proof of

THEOREM 26. Let $\mathfrak{A}$ be a central simple Jordan algebra over $\mathfrak{F}$. Then $\mathfrak{A}$ has a reduction field $\Omega$ of finite degree over $\mathfrak{F}$. 
For we may express the unity element $e$ of $\mathfrak{A}$ as a sum of $q$ pairwise orthogonal primitive idempotents $u_{i}$. If $u_{i} \mathfrak{A} u_{i}$ does not have order one for every $i$ we apply Lemma 14 to obtain a scalar extension $\Omega_{1}$ of finite degree over $\mathfrak{F}$ such that some $u_{i}$ of our sum is not primitive in $\mathfrak{U}_{\Omega_{1}}$. Then we may express $e$ as a sum of $q_{1}>q$ primitive idempotents of $\mathfrak{A}_{\Omega_{1}}$. This process must terminate since the rank of our primitive idempotents decreases at each step. When it terminates we shall have an expression $e=e_{1}+\cdots+e_{t}$ for pairwise orthogonal primitive idempotents $e_{i}$ of $\mathfrak{A}_{\Omega}$ such that $e_{i} \mathfrak{A}_{\Omega} e_{i}$ has order one, $\mathfrak{A}_{\Omega}$ is a reduced algebra.

If $\Re$ and $\mathfrak{R}$ are reduction fields for $\mathfrak{A}$ the degrees of the reduced algebras $\mathfrak{A}_{\boldsymbol{R}}$ and $\mathfrak{A}_{\boldsymbol{R}}$ are the same. Indeed if $u$ is a primitive idempotent of $\mathfrak{A}_{\boldsymbol{\Omega}}$ the algebra $u \mathfrak{A}_{\Re} u$ has order one and so does $u \mathfrak{A}_{\mathfrak{W}} u$ where $\mathfrak{B}$ is the composite of $\Omega$ and $\mathfrak{R}$. But then $u$ is a primitive idempotent of $\mathfrak{A}_{\mathfrak{B}}$. Similarly if $v$ is a primitive idempotent of $\mathfrak{R}$ it is also primitive in $\mathfrak{A}_{\mathfrak{B}}, u$ and $v$ have the same rank $r$, $\mathfrak{A}_{\mathscr{R}}$ and $\mathfrak{A}_{\&}$ have the same degree $t=n / r$.

We may now define the degree of any central simple algebra to be the degree $t$ of $\mathfrak{A}_{\Re}$ for $\Re$ any reduction field $\Re$ of $\mathfrak{A}$. Thus $t$ is an invariant of $\mathfrak{A}$ playing a role similar to that of the degree of a central simple associative algebra.

Reduced algebras play a role for Jordan algebras which is similar to the role of total matric algebras in the theory of associative algebras. Reduction fields are similar to splitting fields. However the roles are not quite the same since the degree of a total matric algebra is its only invariant and this is not the case for reduced algebras. Indeed, we shall prove the existence of two nonisomorphic reduced algebras $\mathfrak{A}$ and $\mathfrak{B}$ over $\mathfrak{F}$ such that $\mathfrak{A}_{\mathfrak{R}}$ and $\mathfrak{B}_{\mathfrak{R}}$ are isomorphic over a suitably chosen quadratic extension $\Re$ of $\mathfrak{F}$.

We pass on now to the determination of all reduced Jordan algebras.

7. Normalization of a reduced algebra. If $\mathfrak{A}$ is any reduced algebra we have seen that every $\mathfrak{A}_{i j}$ contains a quantity $\boldsymbol{e}_{i j}$ such that $\boldsymbol{e}_{i j}{ }^{2}=b_{i i}+c_{j i}$ where $b_{i i}$ and $c_{j i}$ have rank $r$ equal to the rank of $e_{i}$ and $e_{j}$. Then $e_{i j} \rightarrow\left(E_{i j}, E_{j i}\right)$ and, if $\mathfrak{A}$ is a reduced algebra, $E_{i j} E_{j i}=E_{j i} E_{i j}=\lambda_{j} I_{r}$ for $\lambda_{j}$ in $\mathfrak{F}$. Define a matrix

$$
P=\left(\begin{array}{cccc}
E_{11} & & & 0 \\
& E_{12} & & \\
& & \ddots & \\
0 & & E_{1 t}
\end{array}\right),
$$

so that $e_{i} p e_{j}=0$ for $i \neq j$, and let $E_{11}=I_{r}$. We may then replace every $a=\left(A_{i j}\right)$ of $\mathfrak{A}$ by

$$
P a P^{-1}=\left(B_{i j}\right), \quad B_{i j}=E_{1 i} A_{i j} E_{1 j}^{-1} \quad(i, j=1, \cdots, t) .
$$

The mapping $a \rightarrow P a P^{-1}$ is an isomorphism of $\mathfrak{A}$ and the Jordan algebra $P \mathfrak{A} P^{-1}$ such that $e_{i} \rightarrow e_{i}$ for every $i, a_{i j} \rightarrow P a_{i j} P^{-1}$. In particular 


$$
e_{1 j} \rightarrow P e_{1 j} P^{-1}=f_{1 j} \rightarrow\left(F_{1 j}, F_{j 1}\right)
$$

where

$$
F_{1 j}=E_{11} E_{1 j} E_{1 j}^{-1}=I_{r}, \quad F_{j 1}=E_{1 j} E_{j 1} E_{11}^{-1}=\lambda_{j} I_{r} .
$$

Hence we can normalize our algebra $\mathfrak{A}$ so that

$$
e_{1 j} \rightarrow\left(E_{1 j}, E_{j 1}\right), \quad E_{1 j}=I_{r}, \quad E_{j 1}=\lambda_{j} I_{r}
$$

for $\lambda_{j}$ in $\mathfrak{F}$.

If $t>2$ we form the product

$$
e_{i j}=2 e_{1 i} \cdot e_{1 j} \rightarrow\left(E_{i j}, E_{j i}\right)
$$

where

$$
E_{i j}=E_{i 1} E_{1 j}=\lambda_{i} I_{r}, \quad E_{j i}=E_{j 1} E_{1 i}=\lambda_{j} I_{r} .
$$

Hence we have proved that every reduced Jordan algebra $\mathfrak{A}$ may be normalized so that every $\mathfrak{A}_{i j}$ defined for $i \neq j$ contains a quantity $e_{i j} \rightarrow\left(\lambda_{i} I_{r}, \lambda_{j} I_{r}\right)$ where $\lambda_{1}=1, \lambda_{2}, \cdots, \lambda_{t}$ are nonzero quantities of $\mathfrak{F}$.

8. Algebras of degree two. If $\mathfrak{A}$ is any reduced algebra and $i \neq j$ the subalgebra $\left(e_{i}+e_{j}\right) \mathfrak{A}\left(e_{i}+e_{j}\right)$ is a reduced algebra of degree two. Let us then study the properties of such algebras.

We let $a_{12} \rightarrow\left(A_{12}, A_{21}\right)$ be in $\mathfrak{A}_{12}$ and compute $a_{12}{ }^{2}=b_{11}+c_{22}$ where

$$
b_{11} \rightarrow B_{11}=A_{12} A_{21}=f_{1} I_{r}, \quad c_{22} \rightarrow C_{22}=A_{21} A_{12}=f_{2} I_{r}
$$

for $f_{1}$ and $f_{2}$ in $\mathfrak{F}$. Now $B_{11}{ }^{2}=A_{12}\left(A_{21} A_{12}\right) A_{21}=f_{1}{ }^{2} I_{r}=f_{1} f_{2} I_{r}=C_{22}{ }^{2}$ and so $f_{1}=0$ if and only if $f_{2}=0, f_{1}=f_{2}$. Hence

$$
x^{2}=\left(e_{1}+e_{2}\right) f(x)
$$

for every $x$ of $\mathfrak{A}_{12}$ where $f(x)$ is a quadratic form $f\left(\xi_{1}, \cdots, \xi_{s}\right)$ in the coordinates $\xi_{1}, \cdots, \xi_{s}$ of the expression $x=\xi_{1} u_{1}+\cdots+\xi_{s} u_{s}$ of $x$ relative to a basis $u_{1}, \cdots, u_{\diamond}$ of $\mathfrak{Q}_{12}$. The quadratic form may be taken to be reduced and we may write

$$
f(x)=\alpha_{1} \xi_{1}^{2}+\cdots+\alpha_{s} \xi_{s \cdot}^{2}
$$

Then

$$
u_{i} \cdot u_{j}=0, \quad u_{i}^{2}=\alpha_{i}\left(e_{1}+e_{2}\right)
$$

If $t=2$ then $e=e_{1}+e_{2}$ is the unity element of $\mathfrak{A}$ and $\mathfrak{A}$ has a basis

$$
e, u_{1}, \cdots, u_{s+1}=e_{1}-e_{2}
$$

where $u_{i}{ }^{2}=\alpha_{i} e, u_{i} \cdot u_{j}=0$ for every $i \neq j$ and

$$
\alpha_{\varepsilon+1}=1 \text {. }
$$

For $\left(e_{1}-e_{2}\right) \cdot u_{i}=u_{i} / 2-u_{i} / 2=0,\left(e_{1}-e_{2}\right)^{2}=e_{1}^{2}+e_{2}^{2}=e$. 
If $\mathfrak{A}$ is a reduced algebra of degree two and order $s+2$ every $x$ of $\mathfrak{A}$ is a root of a quadratic equation

$$
x^{2}-2 \xi_{0} x+\phi(x) e,
$$

where $\phi(x)=\xi_{0}{ }^{2}+\alpha_{1} \xi_{1}{ }^{2}+\cdots+\alpha_{s} \xi_{s}{ }^{2}+\xi_{s+1}{ }^{2}$ is a nondegenerate quadratic form. Conversely let $\mathfrak{A}$ be any Jordan algebra of order $s+2>2$ with a unity element $e$ and which is such that $\left(x-\xi_{0} e\right)^{2}$ is a nondegenerate quadratic form in the $s+1$ coordinates $\xi_{1}, \cdots, \xi_{s+1}$ of $x$. Then we propose to show that $\mathfrak{A}$ is a central simple algebra of degree two.

Our assumption implies that $\mathfrak{A}$ has a basis $e, u_{1}, \cdots, u_{s+1}$ such that $u_{i} \cdot u_{j}=0$ for $i \neq j$ and $u_{i}{ }^{2}=\alpha_{i}$ e. Adjoin $\left(\alpha_{s+1}\right)^{1 / 2}$ to $\mathfrak{F}$ and see that $v=\left(\left(\alpha_{s+1}\right)^{1 / 2}\right)^{-1} u_{s+1}$ has the property $v^{2}=e$. Then $e_{1}=(e+v) / 2$ and $e_{2}=(e-v) / 2$ are idempotents which are orthogonal and are such that $e_{1}+e_{2}=e$. Thus $\mathfrak{A}_{\Re}$ has a basis $e_{1}, e_{2}, u_{1}, \cdots, u_{s}$. Now $u_{i} \cdot u_{s+1}=\left(u_{i} u_{s+1}+u_{s+1} u_{i}\right) / 2=0, v u_{i}=-u_{i} v$ for $i=1, \cdots, u_{s},(e+v) u_{i}=u_{i}(e-v),(e-v) u_{i}=u_{i}(e+v)$. But then $e_{1} u_{i}=u_{i} e_{2}$, $e_{2} u_{i}=u_{i} e_{1}$ and $e_{1} u_{i} e_{1}=e_{2} u_{i} e_{2}=0$. It follows that $e_{1} x e_{1}=e_{1}\left(\xi_{0} e_{1}+\xi_{s+1} e_{2}+\xi_{1} u_{1}\right.$ $\left.+\cdots+\xi_{s} u_{s}\right) e_{1}=\xi_{0} e_{1}$ and that $e_{2} x e_{2}=\xi_{s+1} e_{2}$ for every $x$ of $\mathfrak{A}$. It remains to show that $\mathfrak{A}$ is simple.

We note that $e_{1} \cdot u_{i}=(e+v) / 2 \cdot u_{i}=u_{i} / 2$ and similarly that $e_{2} \cdot u_{i}=u_{i} / 2$. Let $x=\xi_{0} e_{1}+\xi_{s+1} e_{2}+\xi_{1} u_{1}+\cdots+\xi_{8} u_{s}$ be in an ideal $\mathfrak{B}$ of $\mathfrak{A}$. Then $\mathfrak{B}$ contains $2 e_{1} \cdot x-x=2 \xi_{0} e_{1}+\xi_{1} u_{1}+\cdots+\xi_{s} u_{s}-x=\xi_{0} e_{1}-\xi_{s+1} e_{2}$. But then $\mathfrak{B}$ contains $e_{1} \cdot\left(\xi_{0} e_{1}-\xi_{s+1} e_{2}\right)=\xi_{0} e_{1}$ as well as $-e_{2} \cdot\left(\xi_{0} e_{1}-\xi_{s+1} e_{2}\right)=\xi_{s+1} e_{2}$. If $\xi_{1} \neq 0$ then $\mathfrak{B}$ contains $e_{1}$ and $e_{1} \cdot u_{1}=u_{1} / 2, \mathfrak{B}$ contains $u_{1}{ }^{2}=\alpha_{1} e$ as well as $e, \mathfrak{B}=\mathfrak{A}$. Similarly we may show that $\mathfrak{B}=\mathfrak{A}$ if $\xi_{s+1} \neq 0$. Hence let $x=\xi_{1} u_{1}+\cdots+\xi_{s} u_{s}$ be a nonzero quantity of a nonzero ideal $\mathscr{B}$ and see that $\mathscr{B}$ contains $x \cdot u_{i}=\xi_{i} u_{i}{ }^{2}=\xi_{i} e \neq 0$ for some $i, \mathfrak{B}$ contains $e, \mathfrak{B}=\mathfrak{A}$. We have proved that $\mathfrak{A}$ is simple for every $s>0$.

We have now determined not only all reduced algebras of degree two but all central simple Jordan algebras of degree two. Such algebras have order $s+2$ for $s>0$ and can easily be constructed for every $s$ as subalgebras of a direct product of generalized quaternion algebras.

9. Quadratic forms permitting composition. An involution $J$ of an algebra $\mathfrak{A}$ of order $s$ over a field $\mathfrak{F}$ is a linear transformation $a \rightarrow a^{J}$ over $\mathfrak{F}$ of $\mathfrak{A}$ such that $(a b)^{J}=b^{J} a^{J}$ and $J^{2}$ is the identity transformation. We assume that $\mathfrak{A}$ has a unity element $e$ and that

$$
x+x^{J}=\tau(x) e, \quad x x^{J}=f(x) e
$$

for every $x$ of $\mathfrak{A}$ where $\tau(x)$ and $f(x)$ are in $\mathfrak{F}$. Then $\tau(x)$ is a linear form in the coordinates $\xi_{1}, \cdots, \xi_{s}$ of $x$ relative to some basis of $\mathfrak{A}$ and $f(x)$ is a quadratic form in $\xi_{1}, \cdots, \xi_{s}$.

Assume that $\mathfrak{A}$ is an associative algebra, that $\mathfrak{F}$ is a nonmodular field, and that $f(x)$ is nondegenerate. Then it is known that $s=1$, 2, or 4 . Moreover we may prove that $J$ is unique. For $e^{J}=\tau(e) e-e=\lambda e$ where $\lambda$ is in $\mathfrak{F}$ and $\lambda \neq 0$ 
since $J$ is nonsingular. Then $\left(e^{J}\right)^{2}=(\lambda e)^{2}=\lambda^{2} e=\left(e^{2}\right)^{J}=\lambda e, \lambda^{2}=\lambda, \lambda=1, e^{J}=e$. If $s>1$ every quantity $x$ of $\mathfrak{A}$ is a root of $\lambda^{2}-\tau(x) \lambda+f(x) e=(\lambda e-x)\left(\lambda e-x^{J}\right)$ and $\mathfrak{A}$ has degree two, the minimum function of $\mathfrak{A}$ has degree two and is a polynomial $\phi(\lambda, x)=\lambda^{2}-\alpha(x) \lambda+\beta(x) e$. But $\phi(\lambda, x)$ divides every polynomial with $x$ as a root $\phi(\lambda, x)=(\lambda e-x)\left(\lambda e-x^{J}\right)$ and $x^{J}=\alpha(x) e-x$ is uniquely determined.

If $s=1$ then $\mathfrak{A}=e \mathfrak{F}$ and $e^{J}=e, x=\xi e, f(x)=\xi^{2}$. If $s=2$ then we may always take

$$
f(x)=\xi_{1}^{2}-\alpha \xi_{2}^{2}
$$

and $\mathfrak{A}$ has a basis $e, u$ where $u^{2}=\alpha e \neq 0$. If $\alpha \neq \lambda^{2}$ for any $\lambda$ of $\mathfrak{F}$ then $\mathfrak{A}$ is a quadratic field over $\mathfrak{F}$,

$$
\left(\xi_{1} e+\xi_{2} u\right)^{J}=\xi_{1}-\xi_{2} u
$$

and $J$ is the operation replacing each $x$ of $\mathfrak{A}$ by its conjugate. If $\alpha=\lambda^{2}$ for some $\lambda$ of $\mathfrak{F}$ we may take $\alpha=1, \mathfrak{A}$ has a basis $e_{1}=(e+u) / 2$ and $e_{2}=(e-u) / 2$ and

$$
\stackrel{J}{e_{1}}=e_{2}=\stackrel{2}{e_{2}}, \quad e_{2}^{J}=e_{1}=\stackrel{2}{e_{1}} .
$$

Then $\mathfrak{A}$ is the direct sum of two fields of order one over $\mathfrak{F}$.

Finally let $s=4$. Then $\mathfrak{A}$ is a total matric algebra of degree two over $\mathfrak{F}$ or is a generalized quaternion algebra over $\mathfrak{F}$. In the former case the general element of $\mathfrak{A}$ is a two-rowed square matrix $a$ and $a^{J}$ is the adjoint matrix. Indeed let

$$
a=\left(\begin{array}{ll}
\alpha & \beta \\
\gamma & \delta
\end{array}\right), \quad a^{J}=\left(\begin{array}{rr}
\delta & -\beta \\
-\gamma & \alpha
\end{array}\right)
$$

Then

$$
a+a^{J}=(\alpha+\delta)\left(\begin{array}{ll}
1 & 0 \\
0 & 1
\end{array}\right), \quad a a^{J}=(\alpha \delta-\beta \gamma)\left(\begin{array}{ll}
1 & 0 \\
0 & 1
\end{array}\right) .
$$

We note that

$$
\begin{aligned}
E=\left(\begin{array}{rr}
0 & -1 \\
1 & 0
\end{array}\right), \quad E a^{\prime} E^{-1} & =\left(\begin{array}{rr}
0 & -1 \\
1 & 0
\end{array}\right)\left(\begin{array}{ll}
\alpha & \gamma \\
\beta & \delta
\end{array}\right)\left(\begin{array}{rr}
0 & 1 \\
-1 & 0
\end{array}\right) \\
& =\left(\begin{array}{rr}
-\beta & -\delta \\
\alpha & \gamma
\end{array}\right)\left(\begin{array}{rr}
0 & 1 \\
-1 & 0
\end{array}\right)=a^{J} .
\end{aligned}
$$

Also

$$
b=\left(\begin{array}{ll}
\alpha_{1} & \beta_{1} \\
\gamma_{1} & \delta_{1}
\end{array}\right), \quad a b+(a b)^{J}=\left(\alpha \alpha_{1}+\beta \gamma_{1}+\gamma \beta_{1}+\delta \delta_{1}\right)\left(\begin{array}{ll}
1 & 0 \\
0 & 1
\end{array}\right)
$$


Then if $a \neq 0$ the form $\alpha \alpha_{1}+\beta \gamma_{1}+\gamma \beta_{1}+\delta \delta_{1}$ is not identically zero and is not zero for some $b$. We state this result as

LEMMA 16. If $a \neq 0$ is any two-rowed square matrix there exists a matrix $b$ such that $a b+(a b)^{J} \neq 0$.

10. Reduced algebras of degree $t>2$. Every reduced Jordan algebra $\mathfrak{A}$ of degree $t>1$ has subspaces $\mathfrak{A}_{i j}$ defined for $i \neq j$. We have seen that these subspaces all have the same order $s$ over $\mathfrak{F}$, and that every $a_{i j}$ of $\mathfrak{A}_{i j}$ is associated with a pair of $t$-rowed matrices $A_{i j}, A_{j i}$. Then we have written $a_{i j} \rightarrow\left(A_{i j}, A_{j i}\right)$ and we note that if also $b_{i j} \rightarrow\left(B_{i j}, B_{j i}\right)$ then $\lambda a_{i j}+\mu b_{i j} \rightarrow\left(\lambda A_{i j}+\mu B_{i j}\right.$, $\left.\lambda A_{j i}+\mu B_{j i}\right)$. It follows that the set $\mathfrak{R}_{i j}$ of all matrices $A_{i j}$ is a linear space over $\mathfrak{F}$. Moreover if we employ the normalization of $\S 7$ we see that $2 e_{i j} \cdot a_{i j}$ $=a_{i i}+b_{j i}$ where

$$
a_{i i} \rightarrow A_{i i}=E_{i j} A_{j i}+A_{i j} E_{j i}=\lambda_{j} A_{i j}+\lambda_{i} A_{j i}=\alpha I_{r}
$$

for $\alpha$ in $\mathfrak{F}$, and

$$
b_{j j} \rightarrow B_{j j}=A_{j i} E_{i j}+E_{j i} A_{i j}=A_{i j} \text {. }
$$

Thus $2 e_{i j} \cdot a_{i j}=\alpha\left(e_{i i}+e_{j i}\right)$. Since $\lambda_{i} \lambda_{j} \neq 0$ we see that every $A_{j i}$ is in $\Re_{i j}$, the matrices $A_{i j}$ and $A_{j i}$ all lie in the same linear space $\mathfrak{R}_{i j}$. Indeed let $u_{i j}{ }^{(1)}, \cdots, u_{i j}{ }^{\left({ }^{(}\right)}$be a basis of $\mathfrak{A}_{i j}$ where $u_{i j}{ }^{(k)} \rightarrow\left(U_{i j}{ }^{(k)}, U_{j i}{ }^{(k)}\right)$. Then the matrices $U_{i j}{ }^{(1)}, \cdots, U_{i j}{ }^{(s)}$ span $\mathfrak{R}_{i j}$ but they are not necessarily linearly independent. Also we may take $u_{i j}{ }^{(1)}=e_{i j}, U_{i j}{ }^{(1)}=\lambda_{i} I_{r}, U_{j i}{ }^{(1)}=\lambda_{j} I_{r}, \lambda_{j} U_{j i}{ }^{(k)}$ $=\alpha U_{r}-\lambda_{i} U_{i j}{ }^{(k)}$ and so $U_{i i}{ }^{(1)}, \cdots, U_{j i}{ }^{(e)}$ also span $\Re_{i j}$.

We are assuming that $t>2$ and so have three distinct integers $1, i, j$. Form $2 a_{1 i} \cdot e_{1 j}=b_{i j} \rightarrow\left(B_{i j}, B_{j i}\right)$ where

$$
B_{i j}=A_{i 1} E_{1 j}=A_{i 1}, \quad B_{j i}=E_{j 1} A_{1 j}=\lambda_{j} A_{1 i} .
$$

Evidently $b_{i j}=0$ if and only if $A_{i 1}=A_{1 i}=0$ and so $u_{1 i}^{(1)} \cdot e_{1 j}, \cdots, u_{1 i}^{(s)} \cdot e_{1 j}$ form a basis of $\mathfrak{R}_{i j}$. Moreover it is now evident that $\mathfrak{R}_{1 i}=\mathfrak{R}_{i j}$. By symmetry $\mathfrak{R}_{1 j}=\mathfrak{R}_{i j}$. We have proved that

$$
\mathfrak{R}=\mathfrak{R}_{12}=\mathfrak{R}_{i j}
$$

for every $i \neq j$, that is, all of the matrices $A_{i j}$ of (15) lie in the same linear space $\mathfrak{R}$ of order at most $s$ over $\mathfrak{F}$.

We now form $a_{1 i} \cdot b_{1 j}=c_{i j} \rightarrow\left(C_{i j}, C_{j i}\right)$ where if $a_{1 j} \rightarrow\left(A_{1 i}, A_{i 1}\right)$ and $b_{1 j}$ $\rightarrow\left(B_{1 j}, B_{j 1}\right)$ then

$$
C_{i j}=A_{i 1} B_{1 j}, \quad C_{j i}=B_{j 1} A_{1 i} .
$$

But $A_{1 i}$ and $B_{j 1}$ are completely arbitrary elements of $\mathfrak{R}$ and we have proved that $\&$ is an associative algebra of $r$-rowed square matrices with $I_{r}$ as unity element. We now prove

THEOREM 27. Let the order of $\mathfrak{R}$ be less than $s$. Then $s=2$ and $\mathfrak{A}$ is the total 
matrix algebra of degree $t$ of all rt-rowed square matrices $a=\left(A_{i j}\right), A_{i j}=\alpha_{i j} I_{r}$ for $\alpha_{i j}$ arbitrary elements of $\mathfrak{F}$.

For if the order of $\mathfrak{R}$ is less than $s$ there must exist an $a_{12}$ in $\mathfrak{A}_{12}$ such that $A_{12}=0$ and $a_{12} \neq 0$. Then $A_{21} \neq 0, A_{21}=\alpha I_{r} \neq 0, A_{12}$ contains $f_{21}=\alpha^{-1} a_{12} \rightarrow\left(0, I_{r}\right)$. Also $A_{12}$ contains $e_{12}-\lambda_{2} f_{21}=f_{12} \rightarrow\left(I_{r}, 0\right)$. We form $2 f_{12} \cdot b_{12}$ for any $b_{12} \rightarrow\left(B_{12}, B_{21}\right)$ of $\mathfrak{A}_{12}$ and see that the product is equal to $c_{11}+c_{22}$ where $c_{11} \rightarrow C_{11}=F_{12} B_{21}$ $+B_{12} S_{21}=B_{21}$ since $F_{21}=0, F_{12}=I_{r}$. Then every $B_{21}$ is in $I_{r} \mathfrak{F}, \&$ has order one, $s=2, f_{12}$ and $f_{21}$ form a basis of $\mathfrak{A}_{12}$.

We complete our definition of a basis of $\mathfrak{A}$ by defining $f_{i j}$ to be the $t$-rowed square matrix with the identity matrix in the $i$ th row and $j$ th column and zeros elsewhere. Then $e_{i j}=\lambda_{i} f_{i j}+\lambda_{j} f_{j i}$ and we have already shown that $f_{12}$ and $f_{21}$ form a basis of $\mathfrak{A}_{12}$. We form $2 f_{12} \cdot e_{1 j}=f_{12}\left(\lambda_{2} f_{1 j}+\lambda_{j} f_{j 1}\right)+\left(\lambda_{2} f_{1 j}+\lambda_{j} f_{j 1}\right) f_{12}$ $=\lambda_{i} f_{j 2}$. Similarly $2 f_{21} \cdot e_{1 j}=\lambda_{2} f_{2 j}$ and $\mathfrak{A}_{2 j}$ contains $f_{2 j}$ and $f_{j 2}$, these matrices form a basis of $\mathfrak{A}_{2 j}$. Then $2 f_{12} \cdot f_{2 j}=f_{12} f_{2 j}+f_{2 i} f_{12}=f_{1 j}$ and $2 f_{21} \cdot f_{j 2}=f_{21} f_{j 2}+f_{j 2} f_{21}=f_{i 1}$ form a basis of $\mathfrak{R}_{1 j}$ for every $j \neq 1,2$. Hence $f_{1 j}$ and $f_{j 1}$ form a basis of every $\mathfrak{A}_{1 j}$ for $j>1,2 f_{i 1} \cdot f_{1 j}=f_{i j}, f_{1 i} \cdot f_{1 j}=f_{j i}$ form a basis of $\mathfrak{A}_{i j}$ for every $i \neq j$. We have proved that $\mathfrak{A}$ consists of all $\sum_{i, j} \alpha_{i j} f_{i j}$ for $i, j=1, \cdots, t$ and $\alpha_{i j}$ in $\mathfrak{F}$. This completes our proof.

Let us assume henceforth that $\mathfrak{R}$ has order $s$. This implies that $U_{12}{ }^{(1)}, \cdots, U_{12}{ }^{(8)}$ form a basis of $\mathfrak{R}$ and that $U_{21}{ }^{(1)}, \ldots, U_{21}{ }^{(8)}$ also form a basis of $\&$. Then the mapping $A_{12} \rightarrow A_{21}$ is a nonsingular linear transformation of $R$ and so is the mapping

$$
\mathrm{J}: \quad A_{12} \rightarrow A_{12}^{J}=\bar{A}_{12}=\lambda_{2}^{-1} A_{21} \text {. }
$$

But then if $X$ is any matrix of $\&$ we use (47), (33) to see that

$$
X+\bar{X}=\sigma(x) I_{r}, \quad X \bar{X}=f(X) I_{r},
$$

where $\sigma(x)$ is a linear form in the coordinates of $X$ relative to some basis of $\mathbb{R}$ and $f(X)$ is a quadratic form. Since if $X_{12}+\bar{X}_{12}=\sigma I_{r}$ we have

$$
e_{12}-x_{12} \rightarrow\left(\sigma I-X_{12}, \lambda_{2} \sigma I-\lambda_{2} \bar{X}_{12}\right)=\left(\bar{X}_{12}, \lambda_{2} X_{12}\right)
$$

we see that $\left(X_{12}{ }^{J}\right)^{J}=X_{12}$, that is $J^{2}$ is the identity transformation.

We write $x_{12} \rightarrow\left(X_{12}, \lambda_{2} \bar{X}_{12}\right)$ and form $2 x_{12} \cdot e_{13}=b_{23} \rightarrow\left(B_{23}, B_{32}\right)$ where $B_{23}=X_{21} E_{13}=X_{21}=\lambda_{2} \bar{X}_{12}$ and $B_{32}=E_{31} X_{12}=\lambda_{3} X_{12}$. Then $\lambda_{2} B_{32}=\lambda_{2} \lambda_{3} X_{12}=\lambda_{3} \bar{B}_{23}$. Form $2 e_{12} \cdot y_{13}$ where $y_{13} \rightarrow\left(Y_{13}, Y_{31}\right)$ and obtain $2 e_{12} \cdot y_{13}=c_{23} \rightarrow\left(C_{23}, C_{32}\right)$ where $C_{23}=E_{21} Y_{13}=\lambda_{2} Y_{13}$ and $C_{32}=Y_{31} E_{12}=Y_{31}$. Then $\lambda_{2} Y_{31}=\lambda_{3}\left(\lambda_{2} \bar{Y}_{13}\right)$ and so $Y_{31}$ $=\lambda_{3} \bar{Y}_{13}$. We next form $2 x_{12} \cdot y_{13}=z_{23} \rightarrow\left(Z_{23}, Z_{32}\right)$ where, by (25), $Z_{23}=X_{21} Y_{13}$ and $Z_{32}=Y_{31} X_{12}$. Then $Z_{23}=\lambda_{2} \bar{X}_{12} Y_{13}$ and $Z_{32}=\lambda_{3} \bar{Y}_{13} X_{12}$. It follows that $\lambda_{2} Z_{32}=\lambda_{3} \bar{Z}_{23}=\lambda_{2} \lambda_{3} \bar{Y}_{13} X_{12}=\lambda_{2} \lambda_{3}\left(\bar{X}_{12} Y_{13}\right)^{J}$. Since $\bar{X}_{12}$ ranges over all quantities of $\&$ when $X_{12}$ does we have proved that $\bar{X} \bar{Y}=\bar{Y} \bar{X}$ for all quantities $X$ and $Y$ of $\mathfrak{R}$. Thus $\mathfrak{R}$ satisfies the postulates of our algebras of $\S 9$ and $s=1,2,4$. Note that, by symmetry, $\lambda_{i} A_{j i}=\lambda_{j} \bar{A}_{i j}$.

We have now proved that every reduced Jordan algebra of degree $t>2$ 
can be imbedded in an associative algebra $\mathfrak{S}=\mathfrak{M}_{t} \times \mathfrak{R}$ over $\mathfrak{F}$ where $\mathfrak{M}_{t}$ is the total matric algebra of degree $t$ and $\mathbb{R}$ is one of the algebras of orders one, two or four over $\mathfrak{F}$ of $\$ 9$. We now prove

THEOREM 28. Every reduced Jordan algebra of degree $t>2$ over $F$ is isomorphic to one of the following five types of reduced Jordan algebras:

(1) The Jordan algebra consisting of all t-rowed square matrices with elements in $\mathfrak{F}$.

(2) The Jordan algebra consisting of all J-symmetric t-rowed square matrices $a=a^{J}$ with elements in $\mathfrak{F}$ where we define $a^{J}=P a^{\prime} P^{-1}$ for $a t$-rowed nonsingular diagonal matrix $P$.

(3) The Jordan algebra of all J-Hermitian t-rowed square matrices $a=a^{J}$ with elements in a quadratic field $\Omega$ over $\mathfrak{F}$. Here $\Omega$ has an automorphism $\alpha \rightarrow \bar{\alpha}$ of order two and $a^{J}=P \bar{a}^{\prime} P^{-1}$ for a nonsingular diagonal matrix $P$ with elements in $\mathfrak{F}$.

(4) The Jordan algebra consisting of all $J$-symmetric $2 t$-rowed square matrices $a=a^{J}$ with elements in $\mathfrak{F}$ where we define $a^{J}=P a^{\prime} P^{-1}$ and

$$
P=\operatorname{diag} E_{1}, \cdots, E_{t}=-P^{\prime}, \quad E_{i}=\left(\begin{array}{rr}
0 & -1 \\
1 & 0
\end{array}\right) \text {. }
$$

(5) The Jordan algebra of all J-Hermitian t-rowed square matrices $a=a^{J}$ with elements in a generalized and quaternion division algebra $\mathfrak{D}$. Here $\mathfrak{D}$ has an involution $A \rightarrow \bar{A}$ and $a^{J}=P \bar{a}^{\prime} P^{-1}$ for a nonsingular diagonal matrix $P$ with elements in $\mathfrak{F}$.

For let $\mathfrak{M}_{t}$ have a basis $f_{i j}$ defined for $i, j=1, \cdots, t$ where $f_{i j}$ is then a $t$-rowed square matrix with $I_{r}$ in the $i$ th row and $j$ th column and zeros elsewhere. Every quantity $a$ of $\mathfrak{A}$ can be written in the form $a=\sum_{i, j=1}^{t} A_{i j} f_{i j}$ where $A_{i j}$ is in the associative algebra $\mathfrak{Q}$ with the involution $A \rightarrow \bar{A}$. Define

$$
a^{J}=\sum_{j, i=1}^{t} \lambda_{j}^{-1} \lambda_{i} \bar{A}_{i j} f_{j i}
$$

Then $a b=\left(\sum_{i, j=1}^{t} A_{i j} f_{i j}\right)\left(\sum_{p, q=1}^{t} B_{p q} f_{p q}\right)=\sum_{i, q=1}^{t}\left(\sum_{p=1}^{t} A_{i p} B_{p q}\right) f_{i q}$ and $(a b)^{J}$ $=\sum_{q, i=1}^{t} \lambda_{q}{ }^{-1} \lambda_{i}\left(\sum_{p=1}^{t} \bar{B}_{p q} \bar{A}_{i p}\right) f_{q i}$ since

$$
\overline{A_{i p} B_{p q}}=\bar{B}_{p q} \bar{A}_{i p} .
$$

We also compute $b^{J} a^{J}=\left(\sum_{q, p=1}^{t} \lambda_{q}^{-1} \lambda_{p} \bar{B}_{p q} f_{q p}\right)\left(\sum_{j, i=1}^{t} \lambda_{j}^{-1} \lambda_{i} \bar{A}_{i j} f_{j i}\right)$ $=\sum_{q, i=1}^{t} \lambda_{q}{ }^{-1} \lambda_{i}\left(\sum_{p=1}^{t} \bar{B}_{p q} \bar{A}_{i p}\right) f_{q i}=(a b)^{J}$, and $\left(a^{J}\right)^{J}=\left(\sum_{j, i=1}^{t} \lambda_{j}{ }^{-1} \lambda_{i} \bar{A}_{i j} f_{j i}\right)^{J}$ $=\sum_{i, j=1}^{t} \lambda_{i}{ }^{-1} \lambda_{j} \lambda_{j}^{-1} \lambda_{i} A_{i j} f_{i j}=a$. Thus $a \rightarrow a^{J}$ is an involution of $\mathfrak{M}_{t} \times \mathfrak{R}$ and indeed

$$
a^{J}=P \bar{a}^{\prime} P^{-1}, \quad P=\operatorname{diag}\left\{\lambda_{1} I_{r}, \cdots, \lambda_{t} I_{r}\right\} .
$$

We have also shown that either $\mathfrak{A}=\mathfrak{M}_{t}$ or that every $a=\left(A_{i j}\right)$ where $\lambda_{i} A_{j i}=\lambda_{j} \bar{A}_{i j}$ and hence every $a=a^{J}$. Conversely every $a=a^{J}$ is in $\mathfrak{A}$. 
If $s=1$ then $\mathbb{R}=I_{r} \mathfrak{F}$ and $\mathfrak{A}$ is an algebra of the second type. If $s=2$ and $\mathfrak{Z}$ is a quadratic field over $\mathfrak{F}$ then $\mathfrak{A}$ is an algebra of the third type. If $s=4$ and $\mathbb{R}=\mathfrak{D}$ is a division algebra then $\mathfrak{A}$ is an algebra of the fifth type. If $s=4$ and $\mathbb{R}$ is a total matric algebra we have seen that $\bar{A}=E A^{\prime} E^{-1}$. Then $\mathfrak{A}$ is imbedded in the algebra $\mathfrak{M}_{2 t}=\mathfrak{M}_{t} \times \mathfrak{R}$ of all $2 t$-rowed square matrices and

$$
a^{J}=P a^{\prime} P^{-1}, \quad P=\operatorname{diag}\left\{\lambda_{1} E, \cdots, \lambda_{t} E\right\},
$$

where $a^{\prime}$ is the tranpose of $a$ as a matrix of $\mathfrak{M}_{2 t}$. Now

$$
\begin{aligned}
& Q_{i}=\left(\begin{array}{cc}
\lambda_{i}^{-1} & 0 \\
0 & 1
\end{array}\right), \quad Q_{i} \lambda_{i} E Q_{i}^{\prime}=\left(\begin{array}{cc}
\lambda_{i}^{-1} & 0 \\
0 & 1
\end{array}\right)\left(\begin{array}{cc}
0 & -\lambda_{i} \\
\lambda_{i} & 0
\end{array}\right)\left(\begin{array}{cc}
\lambda_{i}^{-1} & 0 \\
0 & 1
\end{array}\right)=E \\
& =\left(\begin{array}{rr}
0 & -1 \\
1 & 0
\end{array}\right)
\end{aligned}
$$

and if we define $Q=\operatorname{diag}\left\{Q_{1}, \cdots, Q_{t}\right\}$ we see that the set $\mathscr{B}$ of matrices $b=Q a Q^{-1}$ forms a Jordan algebra isomorphic to $\mathfrak{A}$. But $Q a^{J} Q^{-1}$ $=Q P Q^{\prime}\left(Q a Q^{-1}\right)^{\prime}\left(Q P Q^{\prime}\right)^{-1}=P_{0} b^{\prime} P_{0}^{-1}$ where $P_{0}=\operatorname{diag}\left\{E_{1}, \cdots, E_{t}\right\}, E_{i}=E$ as in our theorem.

There remains the case where $\mathfrak{R}$ is an algebra of order two but is not a field. Then the quantities of $\mathfrak{A}$ are imbedded in the direct sum $\left[\mathfrak{M}_{t} \times\left(e_{1}\right)\right]$ $\oplus\left[\mathfrak{M}_{t} \times\left(e_{2}\right)\right]$. Moreover every $a_{i j}$ is a linear combination of $\lambda_{j} f_{i j} e_{1}+\lambda_{i} f_{j i} e_{2}$ and $\lambda_{i} f_{j i} e_{1}+\lambda_{j} f_{i j} e_{2}$. It follows that every

$$
a=\left(\begin{array}{ll}
A & 0 \\
0 & A^{T}
\end{array}\right), \quad A^{T}=P A^{\prime} P^{-1}
$$

where $P=\operatorname{diag}\left\{\lambda_{1} I_{r}, \cdots, \lambda_{t} I_{r}\right\}$. This Jordan algebra is not closed with respect to the associative product since

$$
a b=\left(\begin{array}{cc}
A B & 0 \\
0 & A^{T} B^{T}
\end{array}\right) \neq\left(\begin{array}{cc}
A B & 0 \\
0 & (A B)^{T}
\end{array}\right)
$$

in general. However the mapping $a \rightarrow A$ is a homomorphism of $\mathfrak{A}$ on the Jordan algebra of all $t$-rowed square matrices. Since $a \rightarrow A=0$ if and only if $a=0$ we see that $a \rightarrow A$ is an isomorphism and the Jordan algebras of this type are algebras of type (1).

There remains the proof that our Jordan algebras are simple. For if they are simple they are evidently reduced algebras. Let us then assume that $\mathscr{B}$ is a nonzero ideal of $\mathfrak{A}$ and let $b=\left(B_{i j}\right) \neq 0$ be in $\mathfrak{B}$. By the argument of $\S 3$, $\mathscr{B}$ contains each $b_{i i} \rightarrow B_{i i}$ and each $b_{i j} \rightarrow\left(B_{i j}, B_{j i}\right)$. If some $b_{i j} \neq 0$ then $\mathscr{B}$ contains $b_{i j} \cdot a_{i j}=c_{i i}+c_{j i}$ where $c_{i i} \rightarrow C_{i i}=A_{i j} B_{j i}+B_{i j} A_{j i}$. If $\mathfrak{A}=\mathfrak{M}_{t}$ we take $A_{i j}=1$ and $A_{j i}=0$ if $B_{j i} \neq 0$ and $A_{i j}=0$ and $A_{j i}=1$ if $B_{j i}=0$ so that $B_{i j} \neq 0$. In either case $c_{i i} \neq 0$. If $\mathfrak{R}$ is a division algebra then $b_{i j}{ }^{2}=\lambda_{i} \lambda_{j} f\left(e_{i}+e_{j}\right)$ where $f$ 
is a quadratic form which does not vanish unless $b_{i j}=0$ and so again we have $c_{i i} \neq 0$. The only remaining case is that of the algebra of type (4). In this case we use Lemma 16 and see that we may select $c_{i i}=\lambda_{j} \lambda_{i}^{-1}\left(A_{i j} \bar{B}_{i j}+B_{i j} \bar{A}_{i j}\right) \neq 0$.

We have proved that the ideal $\mathfrak{B} \neq 0$ contains a quantity $b \neq 0$ such that $b_{i i} \neq 0$ for some $i$ and so $\mathfrak{B}$ contains the corresponding $e_{i}$. Then $\mathfrak{B}$ contains $2 e_{i} \cdot\left(\lambda_{j} f_{i j}+\lambda_{i} f_{j i}\right)=\lambda_{j} f_{i j}+\lambda_{i} f_{j i}$ as well as its square $\lambda_{i} \lambda_{j}\left(e_{i}+e_{j}\right)$. It follows that $\mathfrak{B}$ contains every $e_{i}, \mathfrak{B}$ contains the unity element $e$ of $\mathfrak{A}, \mathfrak{B}=\mathfrak{A}$.

11. Split algebras. The role of the total matric algebra in the theory of associative algebras is played in the theory of Jordan algebra by algebras called split algebras. We define such algebras as follows:

(1) The Jordan algebra of all $t$-rowed square matrices. These algebras have degree $t$ and order $t^{2}$.

(2) The Jordan algebra of all $t$-rowed symmetric matrices. These algebras have degree $t$ and order $t(t+1) / 2$.

(3) The Jordan algebra of all $2 t$-rowed matrices $a=a^{J}$ of degree $t>1$ defined by $a^{J}=P a^{\prime} P^{-1}$ where $P$ is skew-symmetric and we may take

$$
P=\operatorname{diag}\left\{E_{1}, \cdots, E_{t}\right\}, \quad E_{i}=\left(\begin{array}{rr}
0 & -1 \\
1 & 0
\end{array}\right) .
$$

The order of the algebra of degree $t$ is $2 t^{2}-t$.

(4) The algebras of degree two with a basis $1, u_{2}, \cdots, u_{s+2}$ where $u_{i}{ }^{2}=1$, $u_{i} \cdot u_{j}=0$ and $s>4$ or $s=3$.

It should be clear that if $\mathfrak{A}$ is any central simple algebra of degree $t$ over $\mathfrak{F}$ and $\Re$ is a reduction field for $\mathfrak{A}$ we can select $\Re$ so that when $\mathfrak{R}$ has order greater than 1 it is not a division algebra. Then we can also select $\Omega$ so that the diagonal matrices $P$ of the algebras of types (2) and (4) of Theorem 28 are the squares of elements of $\Omega$. The replacement of $a$ by $b=Q a Q^{-1}$ replaces $P$ by $Q P Q^{\prime}$ and hence we can always take $P$ to be the identity matrix. It follows that every central simple Jordan algebra $\mathfrak{A}$ of degree $t>2$ has a splitting field $\Omega$ such that $\mathfrak{U}_{\Omega}$ is a split algebra of type (1), (2) or (3).

For algebras of degree $t=2$ we note that, when $s=1, \mathfrak{A}$ contains

$$
\left(\begin{array}{ll}
0 & 1 \\
\lambda_{2} & 0
\end{array}\right)
$$

Then if we adjoin $\mu=\left(\left(\lambda_{2}\right)^{1 / 2}\right)^{-1}$ and replace each $a$ of $\mathfrak{A}$ by $Q a Q^{-1}$ where

$$
Q=\left(\begin{array}{ll}
1 & 0 \\
0 & \mu
\end{array}\right), \quad Q\left(\begin{array}{ll}
0 & 1 \\
\lambda_{2} & 0
\end{array}\right) Q^{-1}=\left(\begin{array}{ll}
0 & 1 \\
\mu^{-1} & 0
\end{array}\right)\left(\begin{array}{ll}
1 & 0 \\
0 & \mu^{-1}
\end{array}\right)=\mu^{-1}\left(\begin{array}{ll}
0 & 1 \\
1 & 0
\end{array}\right) .
$$

Then $\mathfrak{A}_{\boldsymbol{\Omega}}$ is isomorphic to the algebra of all two-rowed square matrices $\Re^{\circ}$ The matrices

$$
I=\left(\begin{array}{ll}
0 & 1 \\
1 & 0
\end{array}\right), \quad u_{2}=\left(\begin{array}{rr}
1 & 0 \\
0 & -1
\end{array}\right)
$$


span the two-rowed total matric algebra $\mathfrak{M}_{2}$ and this Jordan algebra has a multiplication table $u_{i} \cdot u_{j}=0$ for $i \neq j, u_{1}{ }^{2}=u_{2}{ }^{2}=-u_{3}{ }^{2}=I$. Then every Jordan algebra of degree two and order four has a related field $\Omega$ such that $\mathfrak{A}_{\Omega}$ is isomorphic to $\mathfrak{M}_{2}$ over $\Re$. The algebra of all four-rowed $J$-symmetric matrices $a$ defined by

$$
a=\left(\begin{array}{cc}
\xi I_{2} & A \\
E A^{\prime} E^{-1} & \xi I_{2}
\end{array}\right), \quad A=\left(\begin{array}{ll}
\alpha & \beta \\
\gamma & \delta
\end{array}\right), \quad E=\left(\begin{array}{rr}
0 & -1 \\
1 & 0
\end{array}\right)
$$

has the property that

$$
\left(a-\xi I_{4}\right)^{2}=|A| I_{4}
$$

where $|A|=\alpha \delta-\beta \gamma$ is a nondegenerate quadratic form in our four variables. It follows that the reduced algebras of degree two and order six are isomorphic over a splitting field of our split algebra of the third type and order six. Thus the only algebras of degree two not accounted for are the algebras of order $5=s+2$ and order $s+2>6$. Since no two of our split algebras have the same degree and order we have proved

THEOREM 29. Every central simple Jordan algebra $\mathfrak{A}$ over a nonmodular field $\mathfrak{F}$ has an extension field $\Re$ of finite degree over $\mathfrak{F}$ called a splitting field $\left({ }^{12}\right)$ of $\mathfrak{A}$ such that $\mathfrak{A}_{\boldsymbol{R}}$ is a split algebra. Each split algebra is uniquely determined by its degree and order.

The analogue of the Wedderburn theorem for associative algebras states that if $\mathfrak{A}$ is a Jordan algebra over a nonmodular field $\mathfrak{F}$ such that $\mathfrak{A}$ is neither semisimple nor nilpotent then $\mathfrak{A}=\mathfrak{S}+\mathfrak{N}$ where $\mathfrak{N}$ is the radical of $\mathfrak{A}$ and $\mathfrak{S}$ is isomorphic to $\mathfrak{A}-\mathfrak{N}$. The investigation of this theorem requires a knowledge of split algebras. We shall discuss this theorem in a later paper.

The University of Chicago,

Chicago, Ill.

(12) The doctorate dissertation of G. K. Kalisch entitled On special Jordan algebras (Chicago, 1942) consisted mainly of a derivation of the following result: Let $\mathfrak{A}$ be an algebra over a nonmodular field $\mathfrak{F}$ and $\Re$ be a scalar extension of $\mathfrak{F}$ such that $\mathfrak{A}_{\Re}$ is a split algebra of type (2) or type (3). Then there exists an associative simple algebra $\mathfrak{B}$ with an involution $J$ over its center such that $\mathfrak{A}$ consists of all $J$-symmetric quantities of $\mathfrak{B}$. The determination of all simple Jordan algebras then requires only that we determine all algebras $\mathfrak{A}$ such that $\mathfrak{A}_{\Re}$ is a total matric algebra. We conjecture that $\mathfrak{A}$ is necessarily either an associative simple algebra or the set of all $J$-symmetric (Hermitian would be a better term) quantities of an associative simple algebra $\mathscr{O}$ with an involution $J$ inducing an automorphism of order two in the center of $\mathfrak{B}$. 